\title{
Dipeptidyl-peptidase IV (DPPIV/CD26)-activated prodrugs: a succesful strategy for improving water solubility and oral bioavailability
}

Sonsoles Velázquez, ${ }^{\mathrm{a} *}$ Sonia de Castro, ${ }^{\mathrm{a}}$ Alberto Diez-Torrubia, ${ }^{\mathrm{a}}$ Jan Balzarini, ${ }^{b}$ and María-José Camarasa ${ }^{a}$

${ }^{a}$ Instituto de Química Médica (IQM-CSIC), Juan de la Cierva 3, E-28006 Madrid, Spain

${ }^{b}$ Rega Institute for Medical Research, KU Leuven, Minderbroedersstraat 10, B-3000 Leuven, Belgium.

* Address correspondence to this author at the Instituto de Química Médica (IQM-CSIC), Juan de la Cierva 3, E-28006 Madrid, Spain; Tel: +34912587458; Fax: +34915644800; E-mail: sonsoles@iqm.csic.es 


\begin{abstract}
In the search of novel enzyme-based prodrug approaches to improve pharmacological properties of therapeutic drugs such as solubility and bioavailability, dipeptidyl-peptidase IV (DPP IV, also termed as CD26) enzyme activity provides a previously unexplored successful prodrug strategy. This review covers key aspects of the enzyme useful for the design of the CD26-directed prodrugs. The proof-of-concept of this prodrug technology is provided for amine-containing agents by directly linking appropriate di- (or oligo)peptide moieties to a free amino group of a non-peptidic drug through an amide bond which is specifically hydrolized by DPPIV/CD26. Special emphasis will be also made in discussing the design and synthesis of more elaborated tripartite prodrug systems, for further extension of the strategy to hydroxy-containing drugs. The application of this technology to improve water solubility and oral bioavailability of prominent examples of antiviral nucleosides will be highlighted.
\end{abstract}




\section{Graphical abstract}

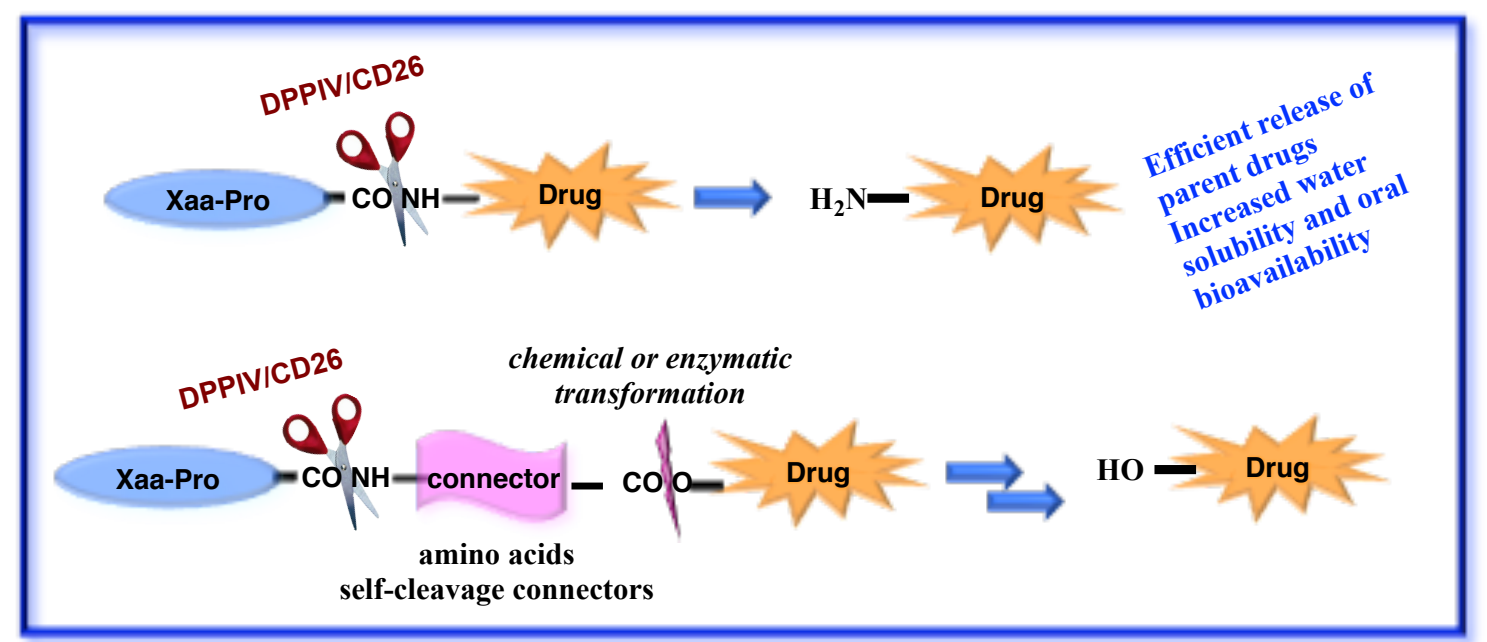

Keywords: Dipeptidyl peptidase IV, CD26 prodrugs, peptides, amine-containing drugs, hydroxy-containing drugs, stability, water solubility, oral bioavailability. 


\section{INTRODUCTION}

Most of the different approaches to design novel drug leads mainly focus on a potent and/or specific drug interaction with an often validated target protein. However, such lead compounds may not have favorable drug-like properties with regard of physicochemical, pharmacokinetic and/or pharmacodynamic properties. Therefore, such candidate drug leads may encounter significant problems during the later steps in clinical drug development.

The term prodrug, designated by Adrien Albert [2] in 1958, mainly refers to biologically inactive derivatives of a drug molecule that require a (in vivo) chemical or enzymatic transformation to release the active parent drug in the body [3, 4]. Simple prodrugs usually contain a covalent bond between a transport moiety or promoiety and the drug molecule (Fig. 1).

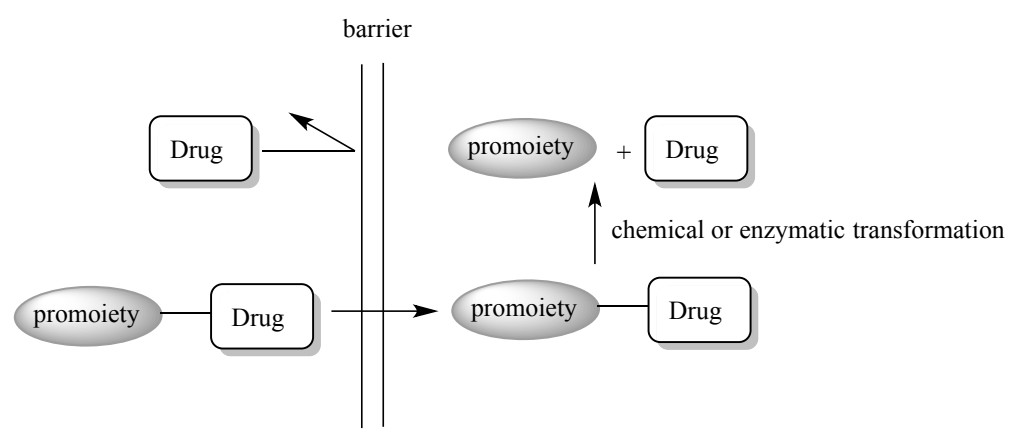

Fig. (1). An illustration of the prodrug concept to overcome or diminish various barriers to drug delivery.

In the process of drug discovery and development, prodrugs represent a useful tool widely used to improve the physicochemical, biopharmaceutical and/or pharmacokinetic properties of pharmacologically active compounds and thus, to enhance the development and utility of a potential drug [5-10]. Thus, prodrugs are designed to mask or avoid an unwanted characteristic of a drug. For example, prodrugs can overcome several obstacles in the formulation and delivery of a drug such as low chemical stability, insufficient aqueous solubility, poor oral absorption, inadequate blood-brain barrier permeability, marked presystemic metabolism and unacceptable toxicity (Fig. (1)) [11-18]. Prodrugs can also be designed to achieve targeted delivery (e.g. brain, liver or tumor targeting) rather than oral bioavailability [19-21]. Their importance is supported by the increasing 
number of approved new drug entities that function, in fact, as prodrugs. Thus, it is now estimated that approximately $10 \%$ of drugs approved worldwide may be categorized as prodrugs and many new prodrug derivatives are currently being investigated $[4,10,13-$ $15]$.

According to the type of drug that has to be released, several prodrug approaches have already been reported. Among them, the classical prodrug approach, which involves enzyme-sensitive covalent bond between the parent drug and a promoiety, represents a well established technology to overcome the problems of poor solubility/bioavailability and toxicity $[3,4,10,16-18]$. Ideally, the promoiety should be non-toxic, easy to synthesize, stable under the conditions of prodrug administration and should undergo easy biodegradation to non-toxic metabolites.

Amino acids have been widely used as promoieties to improve poor aqueous solubility and poor oral bioavailability issues of drugs $[22,23]$. The introduction of amino acids to a parent drug often enhances the water solubility by various orders of magnitude due to the ionized ammonium cation or carboxylate anion present in the polar/ionizable functional groups of the side-chains. Thus, there are several reported examples of amino acid ester prodrugs that have been explored as water-soluble derivatives for oral administration [2427]. Amino acid prodrugs have also led to an increase in permeability, and consequently to an increase in oral bioavailability, by targeting intestinal peptide transporters [28]. Classical examples of amino acid nucleoside prodrugs are the antiherpetic nucleoside analogue prodrugs valacyclovir (Valtrex ${ }^{\circledR}$ ) and valganciclovir (Valcyte ${ }^{\circledR}$ ) [29, 30]. These valyl esters are endowed with a 3-5 fold increase in oral bioavailability. This could be explained by their strong specific interaction with the human peptide transporter hPEPT-1 [31-33] and $\mathrm{ATB}^{0,+}$ transporters $[34,35]$ that seem to recognize (part of) the valine configuration, resulting in the interiorization of the prodrugs in the intestinal cells. Then, cellular esterases are responsable to release the amino acid part (valine) from the prodrug, allowing free parent drug to appear in the blood stream [36]. Other amino acid residues were also used as promoieties, however, the valine appears to have the best combination of carbon chain length and branching at the beta carbon of the amino acid for intestinal absorption. Moreover, the nature of the amino acid promoiety also strongly affects the chemical stability of prodrugs. Steric factors can explain why the addition of a L-valine residue bearing a ramified side chain resulted in a fair, reasonable good chemical stability over the physiological $\mathrm{pH}$ range whereas other amino acids are subjected to 
faster undesirable chemical hydrolysis $[22,37]$. To enhance the chemical stability of the labile ester bond, the amino acid ester prodrugs are generally isolated in their salt forms.

More recently, amino acid prodrugs were also utilized succesfully for sustained release (e.g. D-amphetamine) [38], intravenous administration (e.g. metronidazole [39, 40] and rapamycin [41]) and improve metabolic stability (e.g. gemcitabine prodrugs [42]).

The previous strategies to enhance solubility and oral bioavailability indicate, however, mostly amino acid prodrugs (only one amino acid is attached) of small organic molecules whereby the amino acid is usually coupled to the parental drug through ester bonds, which allows easy conversion back to the free parent drug by esterases. However, there are many ester prodrugs that possess low chemical stability at physiological $\mathrm{pH}$ and it is difficult to predict their rates of bioconversion and, thus, their pharmacological or toxicological effects. On the other hand, attachment of drugs to amino acids or peptides through amide bonds is a strategy much less used due to the high metabolic stability of the amide bond. Unless there is a specific enzyme, the rates of hydrolysis of the prodrugs are generally low and the release does often not take place in vivo at sufficient rate $[3,4]$.

With the aim of exploring innovative enzyme-based prodrug approaches, in the last years a new prodrug technology was reported which can be used to improve the solubility, the bioavailability of therapeutic drugs, or both. The new technology involves the preparation of conjugates of therapeutic drugs with a peptide moiety, so that the conjugate is specifically recognized by the ubiquitous dipeptidyl-peptidase IV (DPP IV) enzyme (Fig. (2)) [43]. This approach can improve the solubility and/or bioavailability of drugs in a potentially more favorable manner since, instead of a single amino acid residue, di- (or oligo)peptide moieties are coupled to a drug molecule (resulting in higher structural diversity and chemical stability). Furthermore, the drug is attached to the di- or oligopeptide via a more chemically stable amide linkage (instead of a more labile ester linkage) which is specifically recognized by the endogenous DPP IV enzyme. The fact that this enzyme is abundantly present in plasma in its soluble form, and in various types of cells, will ensure that eventually the parent drug will be released from the prodrug. It is not expected that the prodrug possesses additional side-effects following conversion to the original drug because a natural, non-toxic peptide moiety would be released. 


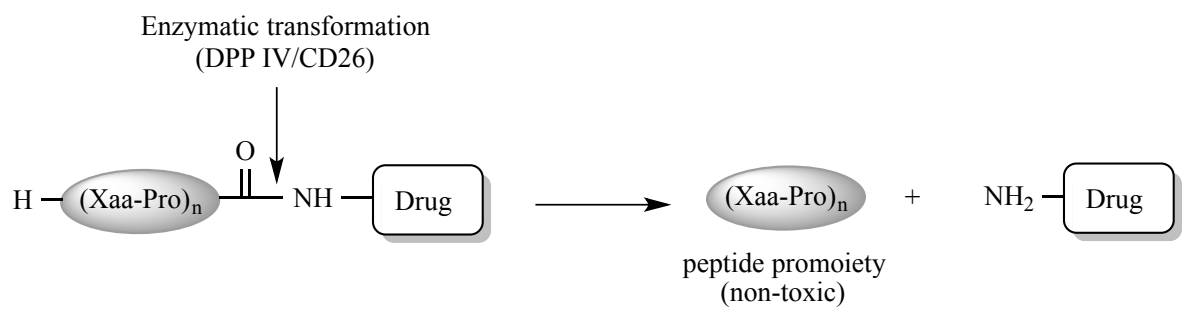

Fig. (2). H-[(Xaa-Pro $\left.)_{n}\right]-[$ drug] prodrugs cleavable by DPP IV/CD26.

This review covers key aspects of the enzyme for the design of the prodrugs, the origin of the prodrug approach and the proof-of-concept of the novel prodrug approach in amine-containing compounds. With the goal of illustrating the scope of the technology, this manuscript also provides the extension of the prodrug approach to hydroxycontaining drug derivatives, and highlights the application of the strategy to improve both water solubility and oral bioavailability of prominent examples of antiviral nucleosides of highly lipophilic nature. Furthermore, practical considerations and challenges associated with the development of DPP IV-based prodrugs will also be discussed. We believe that scientists working in the field of drug discovery and development may find this review as a source of inspiration for drug optimization.

\section{DIPEPTIDYL-PEPTIDASE IV/CD26 ENZYME}

The lymphocyte surface glycoprotein CD26, originally described as a T-cell activation/differentiation marker [44], represents a member of an unique type of membrane-associated peptidases. It features a set of several functional characteristics and it is identical to dipeptidyl-peptidase IV (DPP IV, EC 3.4.14.5) [45-49]. DPP IV/CD26 is classified within the prolyl oligopeptidase family, a group of atypical serine proteinases capable of hydrolyzing a prolyl peptide bond. The 766-amino acid-long enzyme has a short cytoplasmic tail of six amino acids, and it is anchored into the cellular lipid bilayer membrane by a single hydrophobic segment. The membrane anchor to a C-terminal catalytic peptidase domain, a cystein-rich region, and a large extracellular glycosylated area [50]. In mammals this enzyme is widely expressed. It is not only expressed on a variety of subsets of leukocytes (e.g. T cells, B cells, macrophages) but also it is also strongly expressed on epithelial, endothelial and fibroblast cells [45-49]. DPP IV/CD26 also exists as a soluble form present in seminal fluid, plasma and cerebrospinal fluid. This soluble form lacks the intracellular tail and the transmembrane regions [45, 48]. 
This enzyme possesses a dipeptidyl peptidase catalytic activity. It specifically hydrolyzes the amide bond after the penultimate $\mathrm{N}$-terminal amino acid residue (according to Berger and Schechter 1970, P1 residue) of natural peptides [50]. DPPIV/CD26 specifically cleaves X-Pro, or to a lesser degree X-Ala dipeptides from the $\mathrm{N}$-termini of proteins and natural peptides. A well-defined and narrow hydrophobic S1 pocket of the enzyme, bordered by residues Y631, V656, W659, Y662, Y666 and V711, determines this specificity [50]. The side-chain of the N-terminal residue (P2 residue) of the peptide substrate points to a large cavity S2 in the enzyme that gives rise to low specificity for this position. Nevertheless, it is known that this enzyme preferentially recognizes hydrophobic N-terminal residues (e.g. Val) followed by basic (e.g. Arg, Lys), neutral or polar neutral (e.g. Gly, Ser, Gln) and finally showed the least favorable substrate properties in the presence of acidic (e.g. Asp, Glu) residues. A free N-terminus is necessary in order to split-off the dipeptide unit from oligopeptides or polypeptides. Polypeptides containing the third N-terminal amino acid position a proline, hydroxyproline or N-methyl glycine residue are not recognized as substrates by the enzyme [51, 52].

Various cytokines, hematopoietic growth factors, neuropeptides and hormones (with the $\mathrm{X}$-Pro or X-Ala motif at their N-terminal end) are examples of effective substrates for the enzyme $[45,48]$. Interestingly, the presence of a proline at the $\mathrm{N}$-terminus is useful as structural protection against non-specific proteolytic degradation [53]. The DPP IV/CD26 enzyme also efficiently recognizes substrates peptides of different length, from very small natural peptides (e.g. the pentapeptide enterostatin) [54] to larger peptides (e.g. RANTES chemokines SDF-1a and IP-10) of 68- to 77-amino acids [55-57]. The recognition of these peptides by the enzyme results in modulation of their activity, activation, or initiation of degradation depending on the nature of the peptide [48].

It has been described that the tripeptide glycylprolylglycinamide $\left(\mathrm{GPG}-\mathrm{NH}_{2}\right)$ inhibits HIV replication in non-toxic concentrations in cell culture $[59,60]$. Since there was a proline residue in the penultimate amino acid at the amino terminus, it was examined whether GPG-NH${ }_{2}$ could act as a substrate for DPP IV/CD26. It was demonstrated that GPG-NH2 was not active against HIV itself but, instead, acts as a prodrug that need obligatorily hydrolyzed by the enzyme DPP IV/CD26 to glycinamide $\left(\mathrm{G}-\mathrm{NH}_{2}\right)$ for exerting anti-HIV activity in cell culture. It was the first demonstration that a synthetic small molecule GPG- $\mathrm{NH}_{2}$ (antivirally inactive) could be converted to an active antiviral 
drug through the specific action of the DPP IV/CD26 enzyme for the liberation of the active $\mathrm{G}-\mathrm{NH}_{2}$ from the molecule [61].

These results prompted us to investigate whether the presence of DPP IV/CD26 activity in serum/plasma, and on several types of cells, could be used for the design of an entirely novel enzyme-based prodrug technology for improving the solubility and the bioavailability of therapeutic agents or both. Such properties may open entirely new perspectives to implement DPP IV/CD26 activity as a tool for the design of a novel prodrug technology. First interest was given on amine-containing drugs that contain a free amino group that could be directly attached to the carboxyl group of appropriate di(or oligo)peptide promoieties through an amide bond which may then be specifically hydrolyzed by the DPP IV/CD26 enzyme.

\section{APPLICATION OF THE PRODRUG APPROACH TO AMINE-CONTAINING DRUGS}

Human immunodeficiency virus (HIV), the etiological agent of AIDS, mainly infects lymphocytes or macrophages. These types of cells abundantly express DPP IV/CD26 enzyme in their membrane. Thus, anti-HIV compounds were initially investigated, for the validation of this prodrug technology. In particular, attention focused on TSAO derivatives, a unique family of potent and highly specific inhibitors of human immunodeficiency virus type 1 (HIV-1) replication discovered in $1992[62,63]$ in our laboratories in collaboration with the Rega Institute for Medical Research. The prototype compound is $\quad\left[1-\left[2^{\prime}, 5^{\prime}\right.\right.$-bis-O-(tert-butyldimethylsilyl)- $\beta$-D-ribofuranosyl]thymine]-3'spiro-5"-(4"-amino-1",2"-oxathiole-2",2"-dioxide) designated as TSAO-T (1) (Fig. (3)) [63-65]. Given that the reactivity of the 4"-amino group of the spirosultone ring moiety is very low [66] (among the more than 900 TSAO derivatives synthesized over the years [65]), the N-3 aminopropyl TSAO-T derivative (NAP-TSAO-T, 2) (Fig. (3)) [67] was selected as a model amine compound because its primary amine would easily enable the formation of an amide bond with the appropriate peptide promoiety. Moreover, it is known that this TSAO derivative shows similar antiviral activity compared to the prototype TSAO-T [67]. 


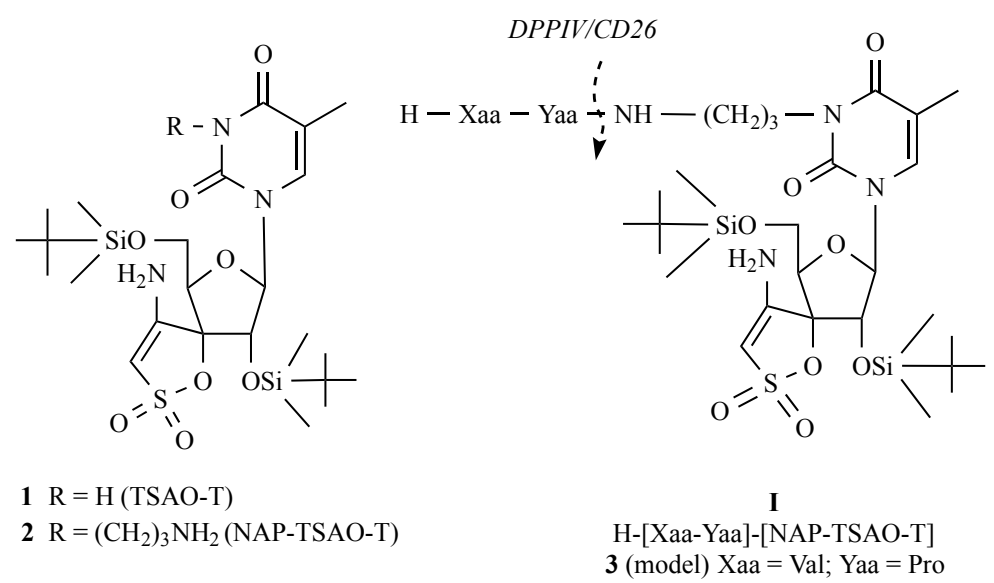

Fig. (3). Structures of TSAO derivatives 1 and $\mathbf{2}$ and H-[Xaa-Yaa]-[NAP-TSAO-T] conjugates of general formula $\mathbf{I}$.

To investigate whether DPP IV/CD26 would recognize and hydrolyze dipeptide sequences linked to a non-peptide molecule, several dipeptidyl amide prodrugs of TSAO molecules of general formula I (Fig. (3)) deprotected at the N-terminus of the peptide were designed as examples of bipartite prodrugs [68]. The Val-Pro dipeptide sequence, easily recognized by the enzyme in natural peptides, was first selected as promoiety on model prodrug 3 (Fig. (3)). The synthesis of the target model conjugate was straightforward by coupling of NAP-TSAO-T 2 with Z-Val-Pro-OH dipeptide under standard coupling conditions (BOP and TEA) followed by deprotection of the $\mathrm{N}$-terminal amino group through catalytic hydrogenation [68]. Stability data of the model prodrug in the presence of purified DPP IV/CD26 revealed that the enzyme recognized the prodrug as an efficient substrate, releasing the parent compound. It was demonstrated that the Nterminus of the dipeptide moiety of the prodrug should be deprotected to act as a substrate of the enzyme since the corresponding Z-protected derivative was completely inactive in this assay. Human serum (HS) and bovine serum (BS) also efficiently hydrolyzed prodrug 3 to release the parent drug. Interestingly, when these stability studies were carried out in the presence of two inhibitors of DPP IV/CD26 (Diprotin A and IlePyr) [69-71] the conversion of prodrug to the parent compound was completely blocked. These data pointed to DPP IV/CD26 as the major enzyme responsible in HS and BS for removing the dipeptide promoiety from the prodrug. These findings showed, for the first time, that dipeptides linked to a non-peptide molecule (model prodrug 3 ) could be efficiently recognized, and hydrolyzed, by the specific action of DPP IV/CD26 enzyme [68]. 
In order to scan the substrate activity requirements for the DPP IV/CD26 enzyme a first set of TSAO prodrugs was prepared. In this series the proline of the model prodrug 3 was maintained and the valine was changed by a wide range of natural amino acids (Xaa) such as hydrophobic (Ala, Phe, Tyr), basic (Lys), neutral (Gly, Asn) or acidic amino acid residues (Asp, Glu) (compounds 4-11, Table 1) [68]. Also, in the second series of prodrugs the valine remained and the proline was replaced by various natural (Ala, Gly, Leu and Phe) and unnatural amino acids such as D-Pro or modified prolines whose substrate specificity is unknown in naturally occurring peptides (compounds 12-19, Table 1). Enzymatic stability studies showed that the reported substrate specificity of DPP IV/CD26 to hydrolyze natural peptides [72] proved also valid when a synthetic compound such as TSAO was attached to a dipeptide. Hydrophobic, aliphatic and basic residues at the amino terminal position (AlaPro and LysPro derivatives $\mathbf{4}$ and 7) were among the most efficient substrates, whereas negatively charged residues such as Asp (AspPro derivative 11) ranked between the least efficient substrates of DPP IV/CD26. When the proline was replaced by either a natural or an unnatural residue, none of the compounds, except the expected ValAla prodrug 12 and the Val-hydroxyproline prodrug 18, showed significant substrate activity against DPP IV/CD26. Similar results were obtained in HS and BS media.

The cLogP values from the various dipeptidyl prodrugs of NAP-TSAO-T revealed that the lipophilicity could also be substantially modulated according to the nature of the Nterminal amino acid (Table 1). Therefore, the half-life and the lipophilicity of the dipeptidyl prodrugs could be significantly varied (cLogP between 3.88 and -0.69$)$ by mostly changing the nature of the $\mathrm{N}$-terminal residue of the dipeptide XaaYaa, in function of the particular needs [68].

Table 1. Conversion of H-[XaaYaa]-[NAP-TSAO-T] conjugates to parent compound 2 in the presence of purified DPP IV/CD26 (1.5 mUnits) after 1, 4 or $24 \mathrm{~h}$ of incubation and cLogP values.

\begin{tabular}{|l|l|l|l|l|l|}
\hline Comp. & H-Xaa-Yaa & \multicolumn{3}{|l|}{$\begin{array}{l}\text { Percent of conversion (\%) to parent } \\
\text { compound 2 }\end{array}$} & \multirow{2}{*}{ LogP $^{\mathrm{a}}$} \\
\hline & & $1 \mathrm{~h}$ & $4 \mathrm{~h}$ & $24 \mathrm{~h}$ & \\
\hline $\mathbf{2}$ & - & - & - & - & 2.70 \\
\hline $\mathbf{3}$ (model) & Val-Pro & 37 & 62 & 61 & 3.39 \\
\hline $\mathbf{4}$ & Ala-Pro & - & 81 & 88 & 2.47 \\
\hline $\mathbf{5}$ & Phe-Pro & - & 37 & 58 & 3.88 \\
\hline
\end{tabular}




\begin{tabular}{|l|l|l|l|l|l|}
\hline $\mathbf{6}$ & Tyr-Pro & 43 & 66 & 79 & 3.21 \\
\hline $\mathbf{7}$ & Lys-Pro & - & 85 & 99 & 2.17 \\
\hline $\mathbf{8}$ & Gly-Pro & 5 & 20 & 58 & 2.16 \\
\hline $\mathbf{9}$ & Asn-Pro & - & 27 & 73 & 1.31 \\
\hline $\mathbf{1 0}$ & Glu-Pro & - & 48 & 92 & -0.69 \\
\hline $\mathbf{1 1}$ & Asp-Pro & - & 7.9 & 30 & -0.27 \\
\hline $\mathbf{1 2}$ & Val-Ala & 0 & 6.5 & 35 & 2.73 \\
\hline $\mathbf{1 3}$ & Val-Gly & 0 & 0 & 0 & 2.42 \\
\hline $\mathbf{1 4}$ & Val-Leu & 0 & 0 & 0 & 4.19 \\
\hline $\mathbf{1 5}$ & Val-Phe & 0 & 0 & 0 & 4.15 \\
\hline $\mathbf{1 6}$ & Val-DPro & 0 & 2.2 & 4.2 & 3.39 \\
\hline $\mathbf{1 7}$ & Val-Hyp(Bzl) & - & 0 & 0 & 5.11 \\
\hline $\mathbf{1 8}$ & Val-Hyp & 4.1 & 18 & 43 & 2.51 \\
\hline $\mathbf{1 9}$ & Val-DHP & 0 & 0 & 0 & 2.44 \\
\hline
\end{tabular}

${ }^{\mathrm{a}}$ The cLogP was calculated using the algorihtm available at the ChemDraw 8.0 program.

In a subsequent report [73], a series of tetrapeptide amide prodrugs of NAP-TSAO (Fig. (4)) were synthesized and their ability to act as efficient substrates for both purified enzyme DPP IV/CD26 as well as human and bovine sera was assayed, with the aim of studying the influence of the length and nature of the peptide promoiety in the half-life and the physicochemical properties of the prodrugs.

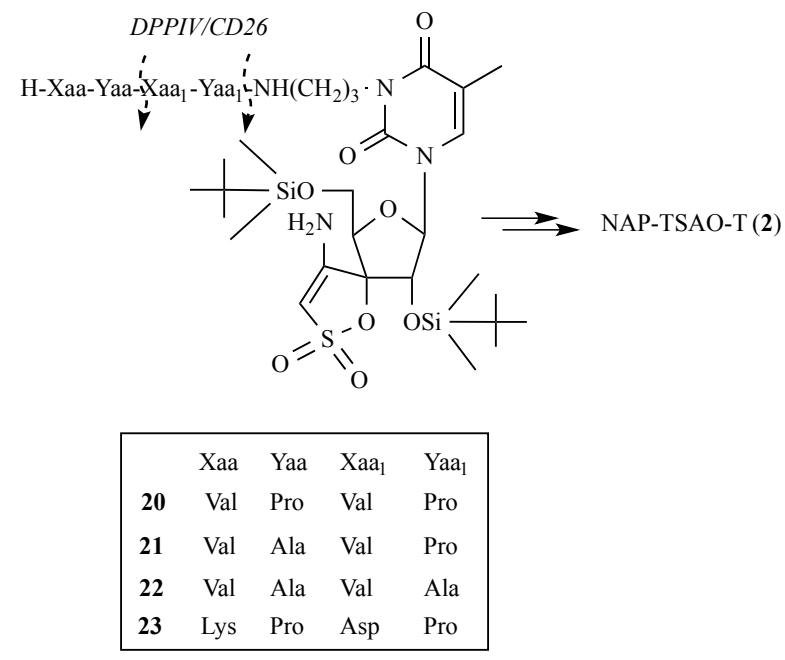

Fig. (4). Structure of tetrapeptidyl TSAO conjugates 20-23, novel two-step activation prodrugs of NAP-TSAO-T in the presence of DPP IV/CD26.

It was shown that tetrapeptide analogues of NAP-TSAO efficiently released the parent compound by DPP IV/CD26 in two successive reaction steps. The conversion efficiency relied on the nature of the amino acids, being the ValProValPro derivative $\mathbf{2 0}$ the more efficient substrate of the enzyme to release the parent compound (more than $80 \%$ 
conversion to the parent compound after $4 \mathrm{~h}$ incubation in the presence of DPP IV/CD26). In the enzymatic conversion reaction, it was not possible to detect dipeptidyl ValPro intermediate from the tetrapeptide prodrug. In contrast, with the LysProAspPro prodrug derivative $\mathbf{2 3}$ the intermediate dipeptide analogue was formed in abundance within 15 min of the reaction after which it was produced a slow hydrolysis to parent derivative (Fig. (5)) [73]. These findings fully agree with the different substrate preferences known for this enzyme (amino-terminal LysPro and ValPro being very efficiently cleaved from the natural peptides and the amino-terminal AspPro being among the worst substrates to be hydrolyzed).

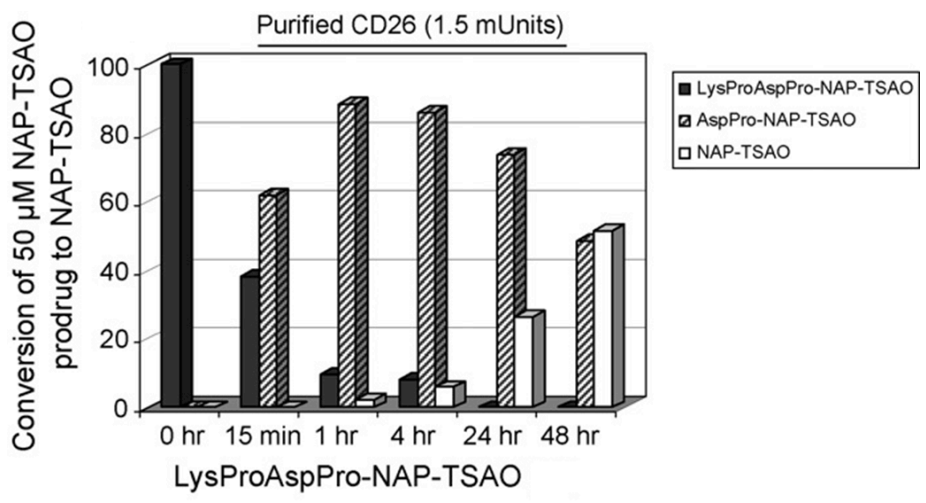

Fig. (5). Conversion of LysProAspPro prodrug derivative 23 to NAP-TSAO-T by purified DPP IV/CD26.

These studies suggest that linking appropriate tetrapeptide moieties allow greater possibilities of structural diversity than dipeptides to modulate the parent compound liberation. In addition, the most hydrophilic LysProAspPro prodrug derivative $\mathbf{2 3}$ enhanced 17 -fold the water solubility $(0.88 \mathrm{mg} / \mathrm{mL})$ compared to the hydrophobic parent derivative $2(0.05 \mathrm{mg} / \mathrm{mL})$ [73]. These findings demonstrated that the DPP IV/CD26based prodrug approach may be useful for improving the water-solubility of lipophilic drugs.

\section{Extension of the prodrug approach to a variety of amine-containing drugs}

Hitherto, the proof-of-concept studies of the DPP IV/CD26 prodrug strategy on TSAO derivatives were carried out by linking di- or tetrapeptide sequences to a primary amino group that was present on an aliphatic chain. Further studies were then conducted on the applicability of the novel peptide-prodrug approach to several amine-containing drugs of different nature (Fig. (6)) [74]. The fluorescent 6-aminoquinoline (6-AQ) [75] which 
carries a primary amino group on an aromatic ring and anthracycline antibiotics such as doxorubicin (Dox) [76, 77], containing a primary amino functional group on a sugar moiety were first selected as examples of an amine-containing aromatic compound or an amino sugar derivative, respectively. In addition, amine-containing drugs with primary amino groups bound to heterocyclic ring systems were also studied. In this context, it was interesting to probe the scope of the approach on nucleoside analogues as they play a key role in antiviral and anticancer therapy. In particular, the lipophilic anti-HIV TSAO cytosine derivative (TSAO- $\mathrm{m}^{5} \mathrm{C}$ ) [78], the polar anticancer cytarabine (ara-C) [79] and the antiviral vidarabine (ara-A) [80-82] drugs were selected as examples of pyrimidine and purine nucleosides containing a primary amino group on the heterocyclic base moiety (Fig. (6)). The target dipeptide conjugates were prepared by coupling the amine drug with the conveniently protected dipeptides followed by $\mathrm{N}$-deprotection. Different coupling conditions and protecting groups were used depending on solubility and reactivity issues of the parent amino-containing compounds [74].

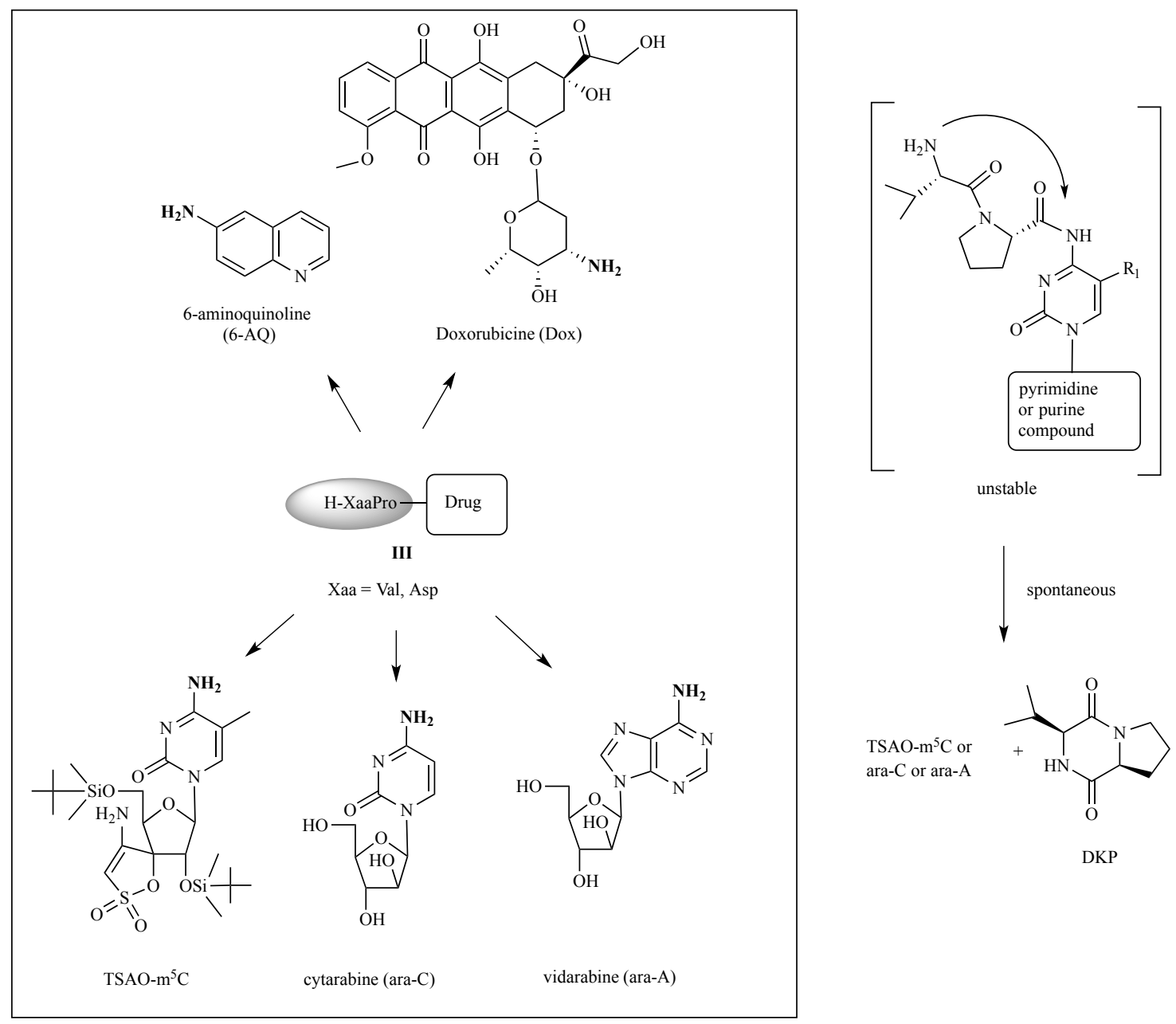

Fig. (6). Structure of target dipeptidyl prodrugs of amine-containing drugs of general formula III. Unstability of dipeptidyl prodrugs of pyrimidine and purine nucleosides due to spontaneous diketopiperazine formation. 
Stability studies demonstrated that the XaaPro dipeptide conjugates (including the Val-Pro model sequence) of 6-AQ and Dox were chemically stable in PBS and efficiently released the parent drug following conversion by purified DPPIV/CD26 as well as by soluble DPPIV/CD26 present in BS and HS [74]. In contrast, when the amino group is located in a pyrimidine or purine heterocyclic (i.e. TSAO- $\mathrm{m}^{5} \mathrm{C}$, cytarabine or vidarabine), the corresponding $\mathrm{N}$-deprotected dipeptide conjugates proved chemically unstable. A spontaneous release of the parent nucleosides and the known diketopiperazine (DKP), due to intramolecular cyclization, was observed during the final $\mathrm{N}$-deprotection step regardless of the deprotection conditions used and of the nature of the dipeptide promoiety (e.g. Val-Pro and Val-Ala) (Fig. (6)) [74]. A significantly lower electrophilicity of the $\mathrm{N}-4$ amide in the cytidine or adenine amide prodrugs may explain why the cytidine and adenine drugs are better leaving groups than the above stable examples of amine-containing drugs in which the amine was present on an aliphatic, aromatic or sugar ring system (NAP-TSAO-T, 6-AQ and Dox). Interestingly, the HValXaaValPro tetrapeptide amide prodrugs of cytidine and adenine nucleoside (Fig. (7)) were far more chemically stable. These prodrugs efficiently released the original drugs by DPPIV/CD26 enzyme in two consecutive steps: first a DPP IV/CD26-catalysed conversion of the tetrapeptide drug to its dipeptide drug derivative, followed by spontaneous release of DKP and parental drug.

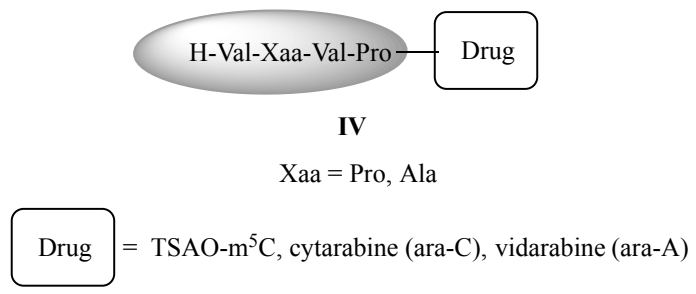

Fig. (7). Structure of chemically stable tetrapeptidyl prodrugs of pyrimidine and purine nucleosides of general formula IV.

In addition, it was also demonstrated that di- or tetrapeptide amide prodrugs of NAPTSAO-T, 6-AQ and vidarabine exhibited (between 5- and more than 60-fold) increased water-solubility compared with the parent compounds irrespective of their lipophilic or polar nature [74].

Thus, these studies showed that a wide range of synthetic drugs/compounds bearing a free amino group on an aromatic, carbohydrate or heterocyclic pyrimidine or purine rings 
can be derivatized with appropriate di- and/or tetrapeptide promoieties, to turn into efficient substrates of DPPIV/CD26 and successfully released the parent drugs in one or two sequential steps. When considering the possibility of applying the DPP IV/CD26based prodrug approach to amine-containing drugs, it is important to notice that di- or tetrapeptides should be used as promoieties depending on the drug's leaving group ability, in order to achieve chemically stable prodrugs [74]. Both the nature and the length of the peptide promoiety permits modulating both the half-life and the lipophilicity of an amine-containing drug as a function of the particular requirements. The prodrug approach proved useful to enhance the aqueous solubility for both lipophilic and polar parent drug molecules.

\section{APPLICATION OF THE PRODRUG APPROACH TO HYDROXY- CONTAINING DRUGS (TRIPARTITE PRODRUGS)}

It would be of utmost importance if the DPP IV/CD26 prodrug strategy could also be extended to hydroxy-containing drugs. As mentioned above, in amine-containing drugs, the amino group of the drug is directly linked to the peptide promoiety through an amide bond specifically recognized and hydrolyzed by DPP IV/CD26 enzyme. In hydroxycontaining drugs the peptide sequence cannot be attached directly to the hydroxyl group through an ester bond because the enzyme cleaved only amide bonds. Thus, more elaborated tripartite conjugates [Xaa-Pro]-[connector]-[drug] had to be designed in this case (Fig. (8)) [83]. In these novel prodrug systems, the presence of a heterobifunctional connector is required to link the hydroxyl group of the drug to the dipeptide promoiety (cleavable by DPP IV/CD26). For the initial studies, the dipeptide ValPro sequence was chosen because it was efficiently hydrolyzed by the enzyme in natural peptides [72] and in previous studies with [peptide]-[drug] conjugates [68, 73, 74]. As heterobifunctional connector, an amino acid was initially chosen to attach the hydroxyl group of the drug to the dipeptide (via a metabolically labile ester linkage). In particular, L-valine was selected since it has been succesfully used as a promoiety for improving the intestinal absorption mediated by the h-PEPT1 transporter (e.g. valacyclovir and valganciclovir) $[29,30]$. Thus, the release of the parent drug from the designed tripartite conjugates occurs through a hydrolysis sequence of two steps: a first enzymatic cleavage by DPP IV/CD26, followed by enzymatic or chemical hydrolysis of the ester bond (Fig. (8)) [83]. The prodrug strategy was applied to several hydroxy-containing drugs bearing different 
types of hydroxyl groups. Thus, the anti-HIV purine nucleoside didanosine (ddI) [84] and acetaminophen (paracetamol) were selected as examples of drugs containing primary hydroxyl groups or hydroxy-containing aromatic compounds. The $\beta$-adrenergic receptor blocker propranolol [85] and the anticancer drug camptothecin [86] were screened as examples of drugs carrying secondary or tertiary hydroxyl groups, respectively (Fig. (8)). The synthesis of these conjugates was carried out by sequential couplings with adequately protected $\mathrm{R}-\mathrm{Val}-\mathrm{OH}$ and $\mathrm{R}-\mathrm{Val}-\mathrm{Pro}-\mathrm{OH}$ followed by deprotection of the amino group. The selection of the strategy of protection and coupling agents depend on the nature of the drug and the reactivity of the different hydroxyl groups in each case [83]. Interestingly, the [ValPro]-[Val]-[drug] prodrugs showed much higher chemical stability in PBS than the valyl intermediates that were often spontaneously converted to the parent drugs. The nature of the hydroxy-containing prodrugs plays a major role in the hydrolysis rate by DPPIV/CD26 and serum. Thus, prodrugs of didanosine (primary hydroxyl group) or acetaminophen (aromatic hydroxyl group) were hydrolyzed more quickly by the enzyme $\left(\mathrm{t}_{1 / 2} 4 \mathrm{~h}\right.$ and $15 \mathrm{~min}$, respectively) than the corresponding camptothecin prodrug (containing a tertiary hydroxyl group). Importantly, some prodrugs (i.e. the campthotecin derivative) showed remarkable increases in water solubility (from 60 - to 205 -fold at $\mathrm{pH}=7.4$ or $\mathrm{pH}=5.0$, respectively) in comparison to the lipophilic and poorly soluble parent drugs [83].

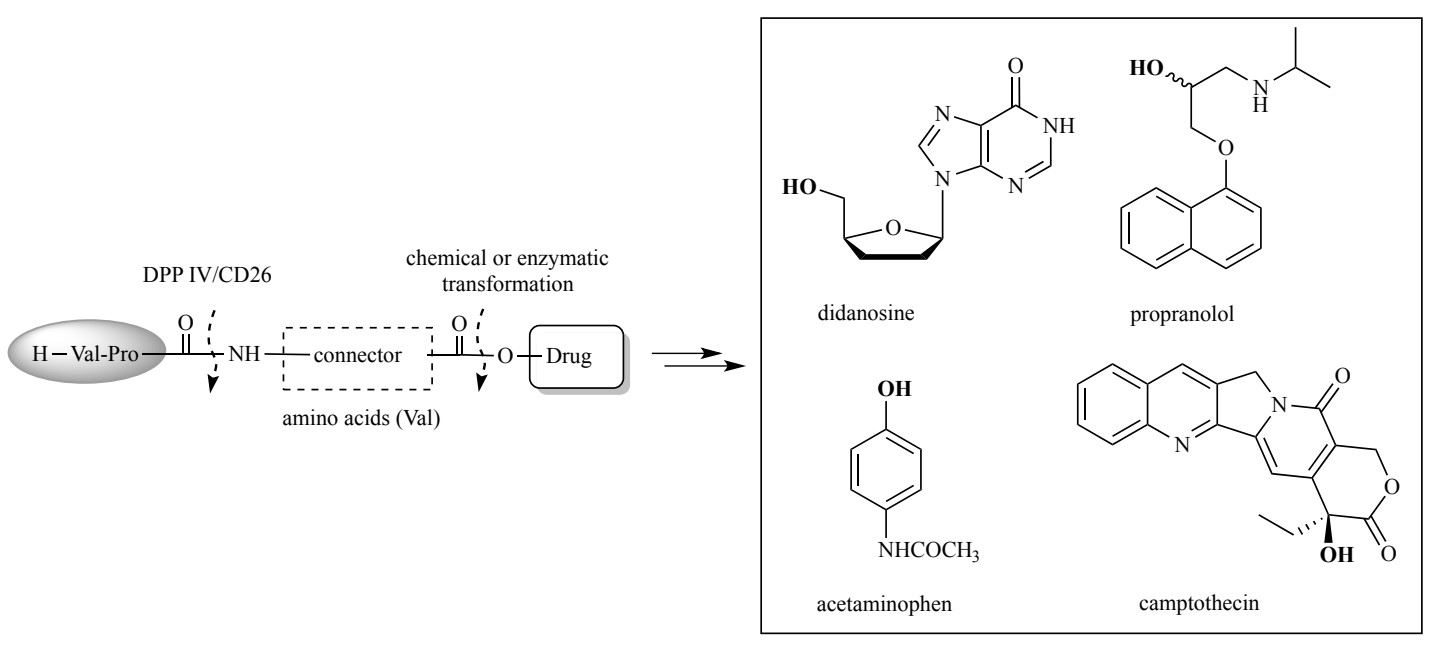

Fig. (8). Design of target tripartite prodrugs based on DPP IV/CD26 enzyme for hydroxy-containing drugs.

These findings showed that the DPP IV/CD26 prodrug technology can be successfully applied to a range of drugs containing hydroxy groups of different nature. Fairly 
chemically stable tripeptide derivatives can be prepared that successfully released the parent drugs [83]. The chemical stability, the susceptibility to hydrolysis by the DPP IV/CD26 enzyme and the final conversion rates to the parent drugs highly depended on the nature of each particular hydroxy-containing drug.

It should also be noted that the chemical stability of the ester bond of the corresponding tripeptide derivatives is much higher than the corresponding amino acid (i.e. valyl) ester prodrugs. Thus, when considering designing a prodrug of hydroxycontaining drugs, the DPP IV/CD26 prodrug technology may be a good choice when the corresponding amino acid (valyl) ester prodrugs proved to be chemically unstable and thus, it would be a useful alternative to the amino acid ester strategy.

\section{Peptidyl amide- versus peptidyl ester prodrugs of acyclovir}

The prodrug approach was also applied to drugs bearing both an amino and a hydroxyl group in the same molecule in order to compare the results of the corresponding peptidyl amide $v s$. ester prodrugs.

The discovery of the acyclic nucleoside guanine analogue acyclovir (ACV) (Fig. (9)) as a selective and potent antiherpetic agent opened the door to a new stage in antiviral chemotherapy [87]. However, ACV showed poor aqueous solubility and also low oral bioavailability [88]. To overcome these problems, several acyclovir prodrugs were previously reported [88-92]. The majority of these prodrugs were compounds acylated at the primary hydroxyl group, such as the widely used L-valine ester prodrug valacyclovir (VACV) (Fig. (9)) [93-95], due to its higher reactivity compared with the 2-amino group of the nucleobase.

To further pursue the DPP IV/CD26 prodrug approach some novel prodrugs of acyclovir (Fig. (9)) were prepared for the following reasons: ${ }^{\text {i) }}$ both the amino and the hydroxyl group may be derivatized in this compound and peptidyl amide versus ester prodrugs could be compared, ii) it will allow to extend the prodrug approach further to guanidine nucleoside drugs with great biological potential and interest and, iii) acylation of the exocyclic amino group of the guanine base with amino acid or peptides had not been described hitherto and the low solubility of this compound together with the intrinsically low nucleophilicity of this type of amino group makes it a difficult synthetic challenge.

The ValPro dipeptide and the ValProValPro tetrapeptide (efficient substrates of the DPPIV/CD26 enzyme) were selected as peptide promoieties for the target peptidyl amide 
prodrugs 21 and 22 (Fig. (9)) [96]. The Val-Pro dipeptide and the valine as connector were chosen as tripeptide promoiety for the peptidyl ester prodrug $\mathbf{2 3}$ based on prior findings [83]. For the preparation of the challenging peptidyl amide prodrugs of acyclovir 21 and 22 both solution-phase and solid-phase synthetic approaches employing a variety of different coupling reagents and resins were examined. The use of acid fluorides as coupling reagents in solution-phase methods and the selection of Ellman's dihydropyran resin in the solid-phase strategy were key issues for the successful synthesis of the target molecules [96]. As in the case of cytidine and adenosine dipeptide prodrugs [74], the Ndeprotected dipeptide amide conjugate of ACV 21 was chemically unstable and could not be isolated due to diketopiperazine formation during the $\mathrm{N}$-deprotection step while the corresponding tetrapeptide amide conjugate $\mathbf{2 2}$ was stable. Chemical stability studies also revealed that in contrast to the valyl ester prodrug VACV, with almost $50 \%$ spontaneous conversion to free ACV, both the tetrapeptide amide prodrug 22 and the tripeptide ester prodrug 23 were completely stable in PBS after 24 h [96].

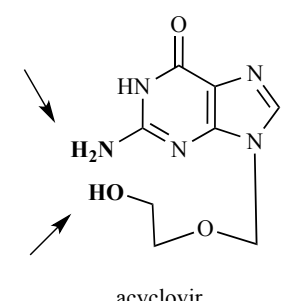

acyclovir

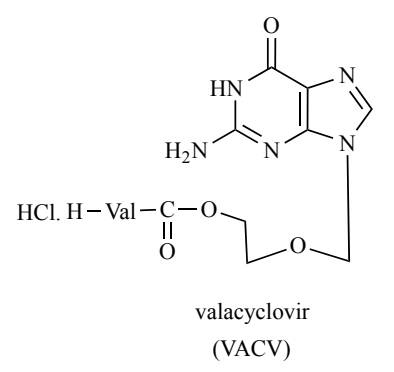

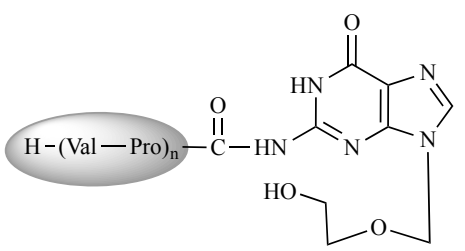

$21(\mathrm{n}=1)$ (chemically unstable)

$22 \quad(\mathrm{n}=2)$

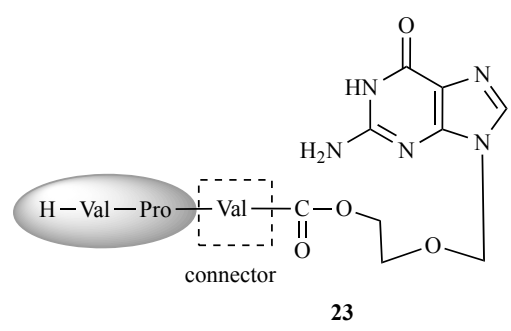

Fig. (9). Structure of acyclovir, valacyclovir and target amide and ester prodrugs 21-23.

Peptidyl amide and ester prodrugs $\mathbf{2 2}$ and $\mathbf{2 3}$ were readily converted to ACV and VACV in the presence of both purified DPP IV/CD26 and bovine and human serum. Interestingly, the tetrapeptide amide prodrug of ACV 22 showed a more rapid hydrolysis to the parent drug in comparison to the tripeptide ester prodrug of ACV $23\left(\mathrm{t}_{1 / 2}<0.25 \mathrm{~h}\right.$ compared to $24 \mathrm{~h}$, respectively). The DPP IV/CD26-catalysed hydrolysis reaction of both prodrugs was effectively inhibited by Vildagliptin, a potent inhibitor of the enzyme. 
Compared to the sparingly soluble parent compound ACV, both amide and ester prodrugs of ACV 22 and $\mathbf{2 3}$ increased significantly the aqueous solubility more than 17-fold and 9fold, respectively [96].

From these studies on ACV prodrugs, cleavable by DPP IV/CD26 using model peptide promoieties, it was concluded that a more rapid hydrolysis to the parent drug can be achieved by targeting peptidyl amide instead of peptidyl ester prodrugs when both amino and hydroxyl functionalities are present on a particular therapeutic drug. Besides, the mechanism of release of the parent drugs from peptidyl amide prodrugs solely depends on DPP IV/CD26-mediated hydrolysis while for peptidyl ester prodrugs a combination of DPP IV/CD26 hydrolysis and subsequent chemical and/or esterasedirected cleavage of the ester bond is required. The chemical stability profile of both amide and ester prodrugs cleavable by DPP IV/CD26 would be higher than that of the corresponding amino acyl (valyl) ester prodrugs.

\section{DPP IV/CD26-DIRECTED PRODRUGS OF THE ANTI-VARICELLA ZOSTER VIRUS AGENT Cf1743 WITH MARKEDLY IMPROVED WATER SOLUBILITY AND ORAL BIOAVAILABILITY}

In the previous examples, the DPP IV/CD26-directed prodrug technology turned out to be effective in improving water solubility of both lipophilic and polar compounds/drugs. Given that a L-valine moiety is implicated in this prodrug strategy, it was important to reveal whether the prodrug technology could also be useful to increase oral bioavailability of therapeutic agents.

Bicyclic furanopyrimidine nucleoside analogues (BCNAs) constitute an interesting class of antiviral compounds which are extremely potent against chickenpox-shingles (VZV) infections [97]. The p-pentylphenyl BCNA analogue (Cf1743, Fig. (10)) is the prototype compound of this family and represents the most selective anti-VZV agent hitherto described with very low toxicity, if any [98, 99]. However, the poor water solubility and limited oral bioavailability compromised the development of the clinical potential of this highly lipophilic compound. 


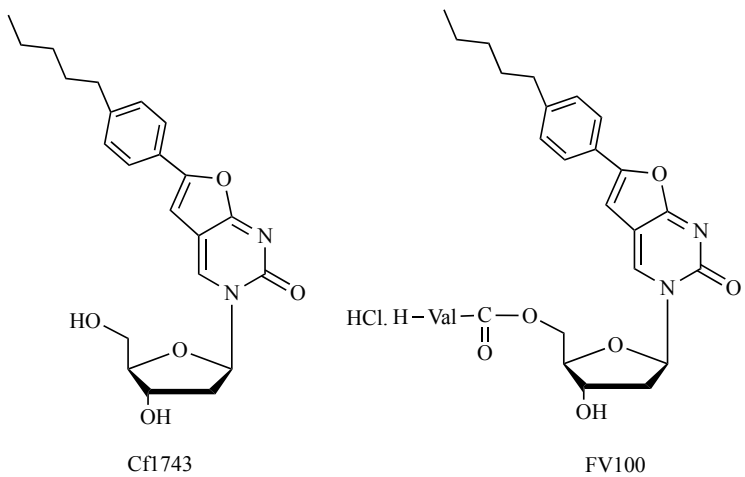

Fig. (10). Chemical structure of the bicyclic pyrimidine nucleoside Cf1743 and its 5'-valyl ester derivative FV100.

Formulation studies with captisol greatly increased the water solubility but did not improve the oral bioavailability [100]. Given the well-established efficacy of 5'-valyl prodrugs of nucleosides, 5'-valyl ester prodrugs of Cf1743 were prepared. Stability studies highlighted the need for the terminal amino group of the valyl prodrug to be in its salt form rather than as its free amino form for the prodrug to be chemically stable. The hydrochloride salt of the 5'-L-valyl ester, named as FV-100 (Fig. (10)), showed a significant improvement of both water solubility and oral bioavailability together with a promising chemical and metabolic stability profile [100]. FV-100 has now entered phase II clinical trials for VZV singles.

The DPP IV/CD26 technology was next applied to this interesting antiviral nucleoside, with the purpose of improving their physicochemical and pharmacokinetic properties.

\section{Prodrugs of Cf1743 with amino acids as connectors}

Cf1743 represents an example of hydroxy-containing nucleoside compounds. Based on previous results, a broad variety of tripeptide prodrugs [XaaPro]-[Yaa]-[Cf1743] (Fig. (11)) cleavable by DPP IV/CD26 enzyme were first designed and prepared [101]. Different sites of esterification of the hydroxyl groups of the nucleoside with peptide promoieties were explored (i. e. 5'- or 3'-monoester and 3', 5'-diester prodrugs). A variety of natural amino acids (Yaa) as connectors and XaaPro dipeptide sequences were also introduced (Fig. (11)). In all cases, the N-deprotected tripeptide prodrugs were isolated in the free amino form. 


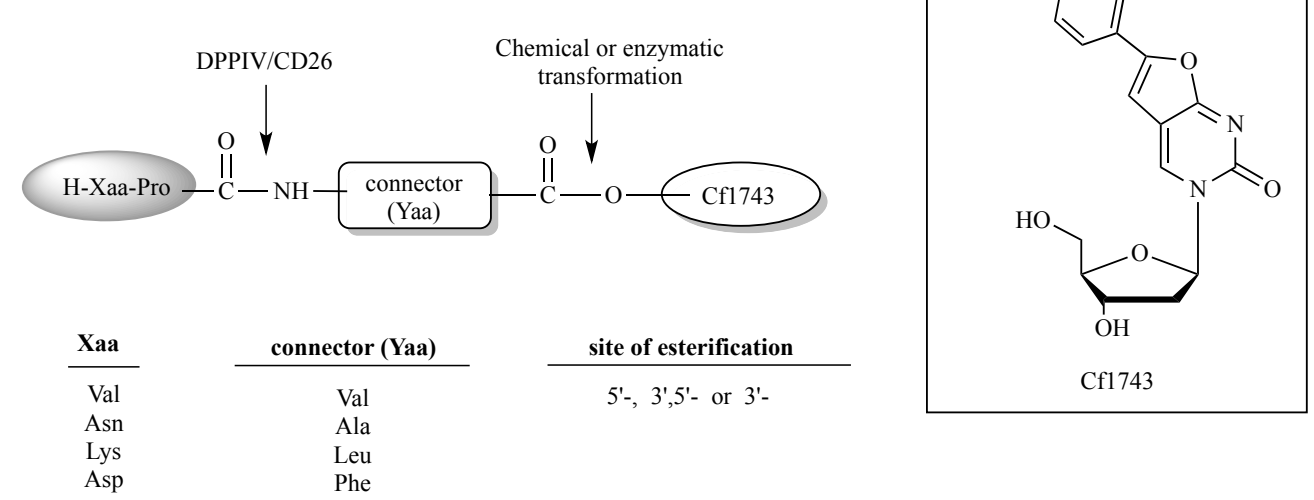

Fig. (11). Design of DPP IV/CD26-cleavable prodrugs of the bicyclic nucleoside Cf1743.

Chemical stability data in PBS indicated that all the tripeptide prodrugs containing a Val as connector proved fully stable after $24 \mathrm{~h}$. However, markedly lower chemical stability of the ester linkage was detected when the Val residue was replaced by Leu, Phe or Ala residues as connectors (Table 2). Enzymatic stability data revealed that the parent Cf1743 compound was efficiently regenerated from the prodrugs in the presence of purified DPP IV/CD26 or by its soluble homologue present in murine, bovine, and human sera. A fine tuning of the half-life of the prodrugs is obtained through the modulation of the nature of the N-terminal residue, in agreement with the earlier observations in other (XaaPro) $)_{n}$ prodrugs $[68,74]$. As expected, all novel prodrugs of Cf1743 exhibited a remarkable enhancement of the aqueous solubility (ranging from 64- to 4294-fold solubility increase) in comparison with the sparingly soluble parent compound (0.018 $\mathrm{mg} / \mathrm{mL}$ ) (Table 2). In general, prodrugs containing polar N-terminal residues such as Asn (30), Lys (31) and Asp (32) turned out to be the compounds with higher water solubility. Notably, the increase in solubility of the novel prodrugs was even higher than that of the reported hydrochloride salt of the 5'-valyl derivative FV100 (ca. 540-fold) [100]. 
Table 2. Aqueous solubility and chemical stability of prodrugs H-[Xaa-Pro]-[Yaa][Cf1743] compared with the parent drug.

\begin{tabular}{|c|c|c|c|c|c|c|c|}
\hline Compd. & Xaa & $\begin{array}{l}\text { Yaa } \\
\text { (connector) }\end{array}$ & $\begin{array}{l}\text { Site of } \\
\text { esterification }\end{array}$ & $C \log \mathrm{P}^{\mathrm{a}}$ & $\begin{array}{l}\text { Aqueous } \\
\text { solubility } \\
(\mathrm{mg} / \mathrm{mL})^{b}\end{array}$ & $\begin{array}{l}\text { Fold } \\
\text { increase } \\
\text { solubility } \\
\text { c }\end{array}$ & $\begin{array}{l}\text { Chemical } \\
\text { stability } \\
(\% \\
\text { remaining } \\
\text { at } 24 \mathrm{~h})^{\mathrm{d}}\end{array}$ \\
\hline Cf1743 & & & & 2.48 & 0.018 & & \\
\hline 24 & Val & Val & 5 & 2 & 1.2 & 68 & $>95 \%$ \\
\hline 25 & Val & Val & $3^{\prime}, 5^{\prime}$ & 1.77 & 77 & 4294 & $>95 \%$ \\
\hline 26 & Val & Val & $3^{\prime}$ & 1.97 & 5.2 & 290 & $>95 \%$ \\
\hline $27^{\mathrm{e}}$ & Leu & Val & 5 & 2.12 & 14 & 766 & $69 \%$ \\
\hline $28^{\mathrm{e}}$ & Phe & Val & 5 & 2.53 & 1.1 & 64 & $52 \%$ \\
\hline $29^{\mathrm{e}}$ & Ala & Val & 5 & 1.63 & 6.1 & 337 & $74 \%$ \\
\hline 30 & Val & Asn & 5 & 1.06 & 39 & 2186 & $>95 \%$ \\
\hline 31 & Val & Lys & 5 & 0.41 & 55 & 3061 & $>95 \%$ \\
\hline 32 & Val & Asp & 5 & 1.3 & 15 & 838 & $>95 \%$ \\
\hline
\end{tabular}
(www.vcclab.org/lab/alogps/)

${ }^{\mathrm{b}}$ Thermodynamic water solubilities measured at $25^{\circ} \mathrm{C}$ after $24 \mathrm{~h}$. The values are mean values of two measurements; variations $<20 \%$.

${ }^{\mathrm{c}}$ Fold-enhancement in aqueous solubility in comparison with the parent compound Cf1743.

${ }^{\mathrm{d}}$ Chemical stability in PBS buffer at $\mathrm{pH}=7.4$ as determined by HPLC at $25^{\circ} \mathrm{C}$ after $24 \mathrm{~h}$.

${ }^{\mathrm{e}}$ Due to reduced chemical stability of these prodrugs, the water solubility was measured after 4 $\mathrm{h}$ instead of $24 \mathrm{~h}$.

Interestingly, a significant increased oral bioavailability in comparison with the parent compound was observed when in vivo experiments in mice were carried out with the 5'ValProVal prodrug derivative 24 (Fig. (12)). It should be noted that its bioavailability was also higher than that of the 5'-Val intermediate prodrug [101].

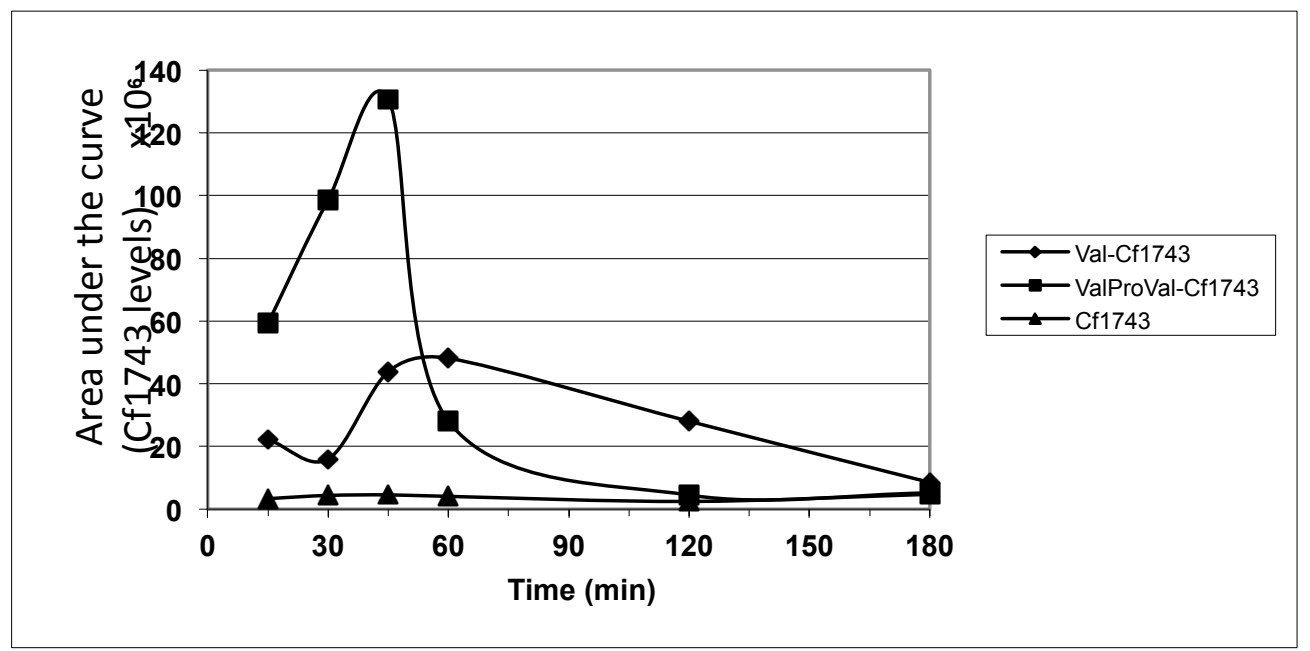

Fig. (12). Cf1743 plasma levels after oral gavage of 5'-Val-Pro-Val prodrug, 5'-Val intermediate and parent drug to mice. 
Oral bioavailability of other selected tripeptide prodrugs was also remarkable improved in comparable levels versus parent drug (Table 3) [101]. In any case, no valyl intermediates of Cf1743 or intact prodrug were detected in the plasma of the exposed mice which demonstrated an efficient in vivo regeneration of the parent drug.

Table 3. Oral bioavailability of the Cf1743 tripeptide prodrugs versus parent compound in mice.

\begin{tabular}{ll}
\hline Compound & Fold-increase of Cf1743 levels in plasma $^{\mathrm{a}}$ \\
\hline 24: 5' '[Val-Pro]-[Val]-Cf1743 & 13 \\
25: 3',5'-di[Val-Pro]-[Val]-Cf1743 & 12 \\
26: 3'-[Val-Pro]-[Val]-Cf1743 & 7 \\
27: 5' -[Val-Pro]-[Leu]-Cf1743 & 11 \\
31: 5' -[Lys-Pro]-[Val]-Cf1743 & 15 \\
1: Cf1743 (parent)
\end{tabular}

${ }^{a}$ Seven time points post oral gavage $(15,30,45,60,90,120,180 \mathrm{~min})$ were considered for each compound. The AUC was calculated as the sum of the Cf1743 levels at the 7 time-points.

\section{Prodrugs of Cf1743 with self-cleavage connectors}

Based on these promising results a novel type of prodrugs of Cf1743 containing selfcleavage spacers systems instead of the previously used amino acids were explored [102]. In these prodrugs, final release of the drug would not be performed by esterase-catalyzed hydrolysis of the amino acid ester intermediates. In particular, prodrugs $\mathbf{3 3}$ and $\mathbf{3 4}$ bearing ethylene- and propylenediamine spacers [103-105] linked to the 5-hydroxyl group of the nucleoside through a carbamate bond were first designed. Focus was also given on tetrapeptide ester prodrugs 35a-c incorporating a dipeptide cyclization connector of a different nature (Fig. (13)) [92, 106-110]. These novel prodrugs were designed to liberate the original parent drug following a two-step mechanism: a first step of enzymatic hydrolysis (DPP IV/CD26) followed by a spontaneous cyclization-elimination step to yield a cyclic urea derivative $[103,104]$ or a diketopiperazine $[92,105-109]$. 


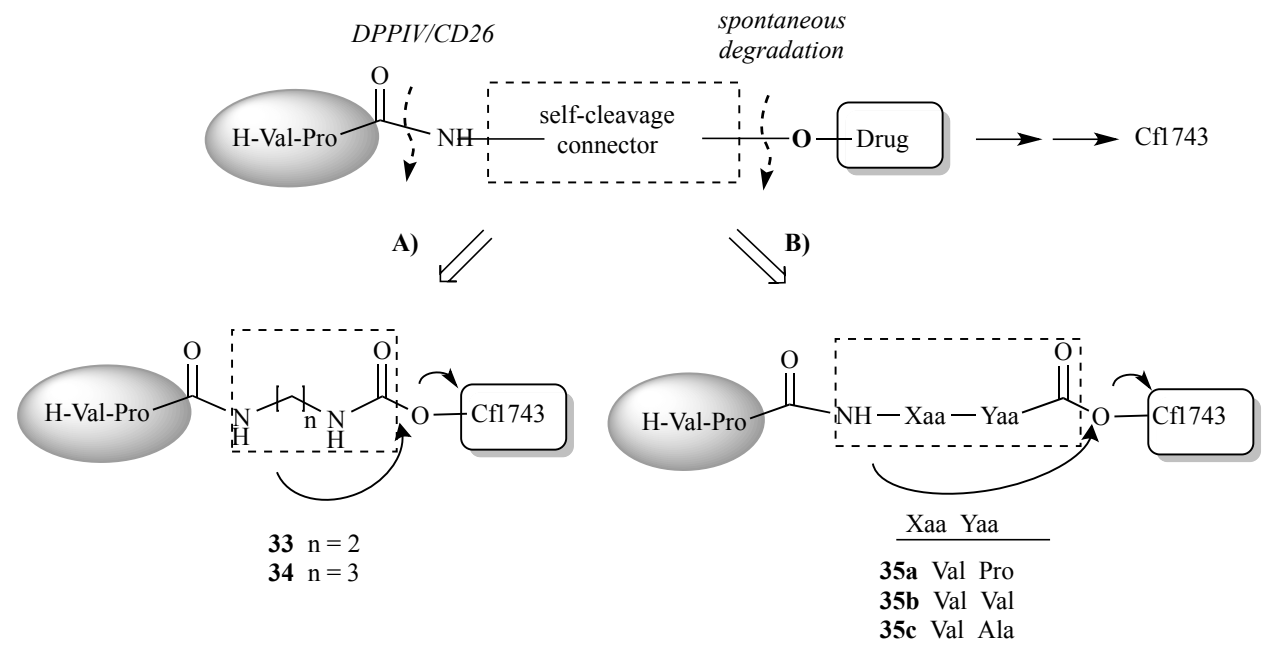

Fig. (13). Design of DPP IV/CD26-activated prodrugs of the anti-VZV drug Cf1743 bearing cyclization self-cleavage connectors.

Both carbamate and tetrapeptide ester prodrugs proved chemically stable in PBS. Enzymatic stability studies, in the presence of purified DPP IV/CD26 and human serum, demonstrated that carbamate prodrugs mainly released their alkyldiamino intermediates. In contrast, the parent nucleoside was efficiently regenerated from the tetrapeptide ester prodrugs after hydrolysis by DPP IV/CD26. The nature of the C-terminal amino acid significantly modulates the rate of drug liberation. Thus, as expected, the ValProValPro prodrug 35a carrying a proline residue at the C-terminal end (which is highly susceptible to cyclization generating the DKP) [110-112], quickly released the parent drug and the ValPro dipeptide intermediate was not observed. However, a slower drug release was observed from the ValProValVal prodrug derivative 35b bearing a bulky C-terminal valine residue, and the ValVal prodrug intermediate was detected during the conversion process even after $24 \mathrm{~h}$.

All novel prodrugs of Cf1743 markedly increased the water solubility (values ranged from 434- to 1804-fold solubility enhancement) as compared with the sparingly soluble parent nucleoside Cf1743 [102]. Moreover, administration of the ValProValVal prodrug 35 to Caco-2 monolayer cell cultures showed the appearance of substantial amounts of the parent drug at the basolateral side of Caco-2 cell cultures. Also, the tetrapeptide prodrug 35 also increased the oral bioavailability of Cf1743 by 15 - to 20 -fold in mice (Fig. (14)) [102]. Notably, the oral bioavailability of the tetrapeptide prodrug of Cf1743 35 was $\geq 2$-fold greater than the oral bioavailability described earlier for the tripeptide prodrugs of Cf1743 [101]. Consequently, both tripeptide and tetrapeptide ester prodrugs 
of Cf1743 may be considered as two new types of effective DPP IV/CD26-based prodrugs with enhanced oral bioavailability and aqueous solubility.

This was an important study, which demonstrates the applicability and utility of the novel DPP IV/CD26 prodrug concept in greatly improving the water solubility and oral bioavailability of (hydrophobic) nucleoside drugs that may be further extended to other poorly soluble and/or poorly oral bioavailable (hydrophobic) drugs.

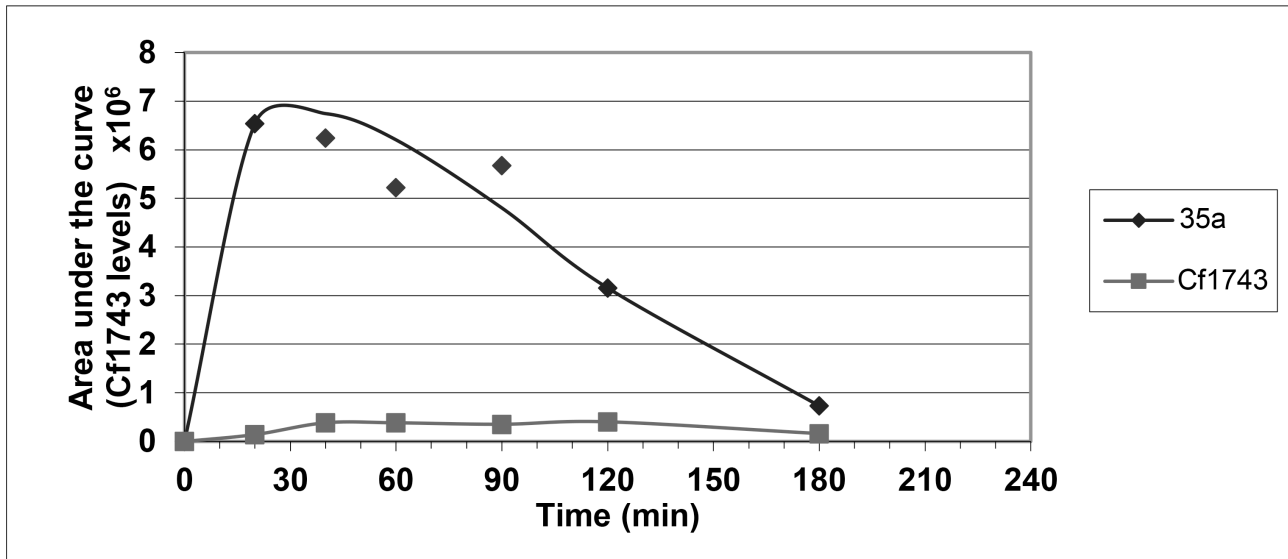

Fig. (14). Administration to mice of the 5'-Val-Pro-Val-Pro prodrug of Cf1743 and parent drug by oral gavage.

\section{CONCLUSIONS AND REMARKS}

Prodrugs continue being an exciting field of research. The examples discloses in this review illustrate the first investigations on a novel appealing prodrug concept. The strategy involves the coupling of a therapeutic drug to di- or oligopeptides, so the $\mathrm{N}$ deprotected peptide conjugates are converted in an efficient manner to the parent compounds specifically by the action of the DPP IV/CD26 enzyme, a ubiquitous enzyme in mammalians.

The therapeutic agents/compounds that were used in the proof-of-concept of this novel technology include a variety of amine- and hydroxy-containing compounds. For aminecontaining drugs, the free primary amino group may be attached directly to the carboxyl group of the di- or tetrapeptide moiety through an amide bond that would be hydrolyzed by DPP IV/CD26. Tetrapeptide amino acid sequences as promoieties can be very useful when the corresponding dipeptide amide prodrugs proved to be chemically unstable (i.e. in case of pyrimidine and purine nucleosides). For hydroxy-containing drugs, the dipeptide and the therapeutic drug should be coupled via a connector that can be an 
amino acid (preferably a valine residue for chemical stability issues) or a self-cleavage linker (preferably a dipeptide cyclization connector).

The benefits of this prodrug approach are the following: ${ }^{i)}$ a successful conversion to the parent drug/compound is ensured by the fact that the CD26 enzyme is ubiquitous and abundantly present in plasma and on the membrane of several types of cells; ${ }^{\text {ii) }}$ there is an adequate structural protection against nonspecific proteolytic degradation due to the presence of a proline in the peptide promoiety; iii) a natural, non-toxic peptide promoiety is released, after conversion of the prodrug to its parent compound; ${ }^{\text {iv) }}$ chemically stable amide or ester peptide prodrugs in their free amino form can now be obtained as compared to the less stable amino acid ester prodrugs that need to be in their salt forms for reasonable stability; ${ }^{v}$ a fine-tuning of the half-life and solubility of the prodrug can be achieved through the modulation of the length and the nature of the oligopeptide promoiety in function of the particular needs; ${ }^{\mathrm{vi})}$ remarkable increases in water solubility of both, lipophilic and polar parent compounds, can be pursuit, a property that might be of benefit in drug formulation issues and vii) significant enhancement of oral bioavailability in mice was demonstrated with different tri- or tetrapeptide ester prodrugs cleavable by DPP IV/CD26 for lipophilic antiviral nucleosides (i. e. Cf1743 nucleoside analogue).

From this study, it can be concluded that the DPP IV/CD26 activity-based prodrug concept is a promising new prodrug technology useful to improve the oral bioavailability and water solubility of therapeutic drugs and thus, to fine-tune the eventual in vivo activity of drugs. However, further work should be needed to fully explore the potential and scope of this approach. We hope this review will stimulate medicinal chemists to implement the DPP IV/CD26-based prodrug strategy to overcome the stability, and solubility and/or bioavailability challenges on novel amino- or hydroxy-containing drugs currently under investigation. 


\section{ABBREVIATIONS}

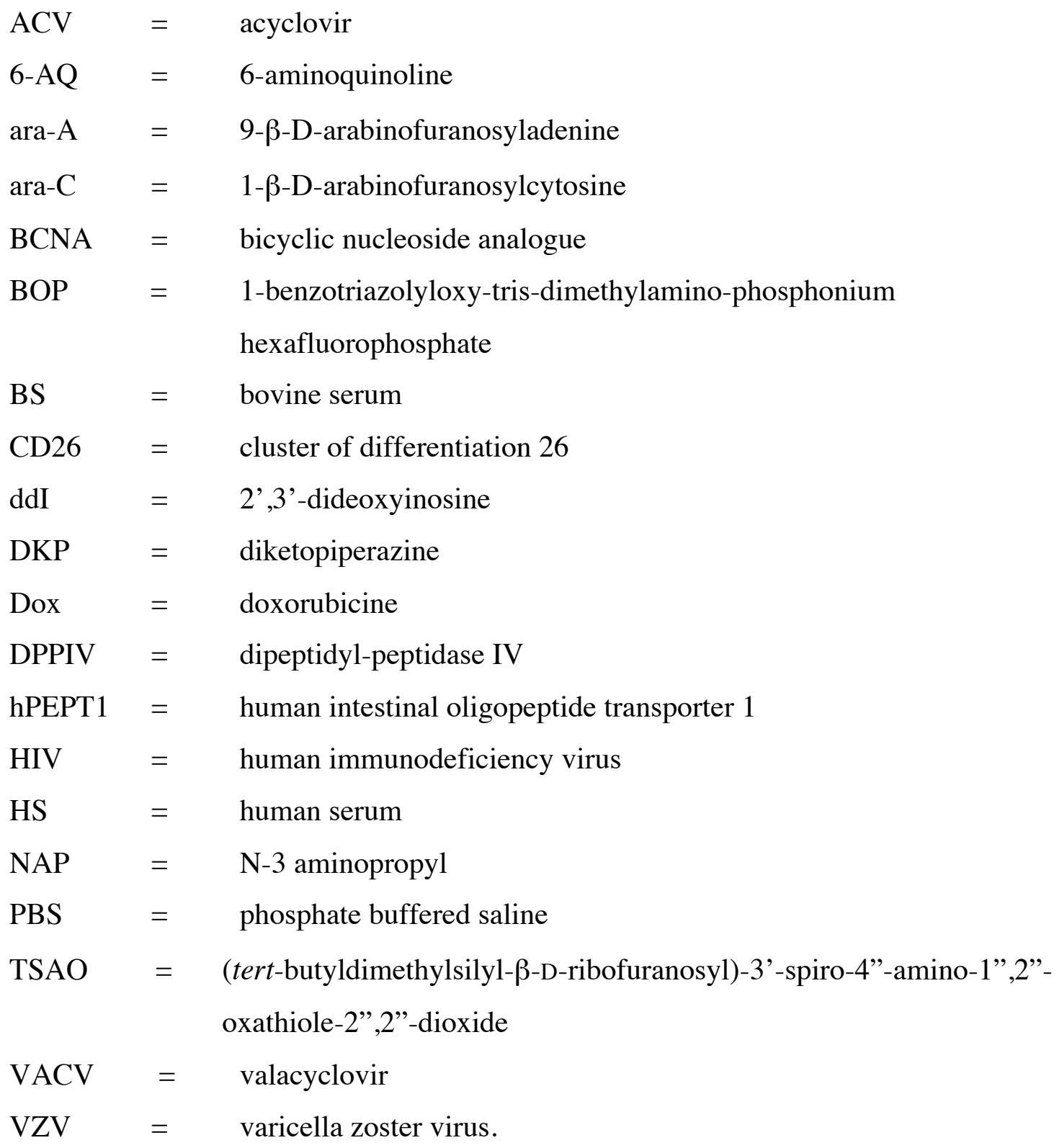

\section{CONFLICT OF INTEREST}

The autor(s) confirm that they have no conflicts of interest with the content of the review. 


\section{ACKNOWLEDGEMENTS}

We thank Ria Van Berwaer and Leentje Persoons for excellent technical assistance. We also thank the Spanish MEC/MICINN (SAF2012-39760-C02), the Comunidad de Madrid (BIPEDD-2-CM ref S-2010/BMD-2457), and the KU Leuven (GOA No. 10/014) for financial support. A Juan de la Cierva contract to S.d.C. (JDC-MICINN) from the Spanish Ministry of Science and Innovation is also gratefully acknowledged.

\section{REFERENCES}

[1] Borchardt, R. T.; Kerns, E. H.; Hageman, M. J.; Thakker, D. R.; Stevens, J. L. Optimizing the "Drug-Like" properties of leads in drug discovery; AAPS Press/Springer: New York, 2006.

[2] Albert, A. Chemical aspects of selective toxicity. Nature, 1958, 182, 421-422.

[3] Stella, V. J.; Borchardt, R. T.; Hageman, M. J.; Oliyai, R.; Maag, H.; Tilley, J. W. Prodrugs: challenges and rewards; Parts 1 and 2; AAPS Press/Springer: New York, 2007.

[4] Wermuth, C. G. Designing prodrugs and bioprecursors. In The practice of medicinal chemistry. Wermuth C. G. Ed., second edition, Academic Press, London 2003; pp 561-582.

[5] Rautio, J.; Kumpulainen, H.; Heimbach, T.; Oliyai, R.; Oh, D.; Järvinen, T.; Savolainen, J. Prodrugs: design and clinical applications. Nat. Rev. Drug Discov., 2008, 7, 255-270.

[6] Ettmayer, P.; Amidon, G. L.; Clement, B.; Testa, B. Lessons learned from marketed and investigational prodrugs. J. Med. Chem. 2004, 47, 2393-2404.

[7] Stella, V. J. Prodrugs as therapeutics. Expert Opin. Ther. Pat. 2004, 14, 277-280.

[8] Testa, B. Prodrug research: futile or fertile? Biochem. Pharmacol. 2004, 68, 2097 2106.

[9] Testa, B. Prodrugs: bridging pharmacodynamic/pharmacokinetic gaps. Curr. Opin . Chem. Biol. 2009, 13, 338-344.

[10] Zawilska, J. B.; Wojcieszak, J.; Olejniczak, A. B. Prodrugs: a challenge for the drug development. Pharmacol. Reports, 2013, 65, 1-14. 
[11] Jana, S.; Mandlekar, S.; Marathe, P. Prodrug design to improve pharmacokinetic and drug delivery properties: challenges to the discovery scientists. Curr. Med. Chem. 2010, 17, 3874-3908.

[12] Clas, S. D.; Sánchez, R. I.; Nofsinger, R. Chemistry-enabled drug delivery (prodrugs): recent progress and challenge. Drug Discov. Today, 2014, 19, 79-87.

[13] Hsieh, P.; Hung, C.; Fang, J. Current prodrug design for drug discovery. Curr. Pharm. Des. 2009, 15, 2236-2250.

[14] Stella, V. J. Prodrugs: some thoughts and current issues. J. Pharm. Sci. 2010, 99, $4755-4765$.

[15] Huttunen, K. M.; Raunio, H.; Rautio, J.; Prodrugs-from serendipity to rational design. Pharmacol. Rev. 2011, 63, 750-771.

[16] Stella, V. J.; Nti-Addae, K. W. Prodrug strategies to overcome poor water solubility. Adv. Drug Deliver. Rev. 2007, 59, 677-694.

[17] Testa, B. Prodrug objectives and design. ADME-Tox approaches. Testa B., Van de Waterbeemd, H. Ed.; in Comprehensive Medicinal Chemistry II. Elsevier; Oxford, UK, 2007; Vol. 5, pp 1009-1041.

[18] Zhang, Y. X.; Sun, J.; Sun, Y. B.; Wang, Y. J.; He, Z.G. Prodrug design targeting intestinal PepT1 for improved oral absorption: design and performance. Curr. Drug Metabolism, 2013, 14, 675-687.

[19] See for example: Vlieghe, P.; Khrestchatisky, M. Medicinal Chemistry based approaches and nanotechnology-based systems to improve CNS drug targeting and delivery. Med. Res. Rev. 2013, 33, 457-516.

[20] Mahato, R.; Tai, W.; Cheng, K. Prodrugs for improving tumor targetability and efficiency. Adv. Drug Deliv. Rev. 2011, 63, 659-670.

[21] Huttunen, K. M.; Mahonen, N.; Raunio, H. Cytochrome P450-activated prodrugs: targeted drug delivery. Curr. Med. Chem. 2008, 15, 2346-2365.

[22] Vig, B. S.; Huttunen, K. M.; Laine, K.; Rautio, J. Amino acids as promoieties in prodrug design and development. Adv. Drug Deliv. Rev. 2013, 65, 1370-1385, and references therein.

[23] Vig, B. S.; Rautio, J. Amino acid prodrugs for oral delivery: challenges and opportunities. Ther. Deliv. 2011, 2, 959-962.

[24] See for example: Chan, O. H.; Schmid, H. L.; Stilgenbauer, L. A.; Howson, W.; Horwell, D. C.; Stewart, B. H. Evaluation of a targeted prodrug strategy of enhance oral absorption of poorly water-soluble compounds. Pharm. Res. 1998, 15, 1012-1018. 
[25] Marathe, P. H.; Kamath, A. V.; Zhang, Y.; D’Arienzo, C.; Bhide, R.; Fargnoli, J. Preclinical pharmacokinetics and in vitro metabolism of brivanib (BMS-540215) a potent VEGFR2 inhibitor and its alanine ester prodrug brivanib alaninate. Cancer Chemother. Pharmacol. 2009, 65, 55-66.

[26] Gingrich, D. E.; Reddy, D. R.; Iqbal, M. A.; Singh, J.; Aimone, L. D.; Angeles, T. S.; Albom, M.; Yang, S.; Ator, M. A.; Meyer, S. L.; Robinson, C.; Rugeri, B. A.; Dionne, C. A.; Vaught, J. L.; Mallamo, J. P.; Hudkins, R. L. A new class of potent vascular endotelial growth factor receptor tyrosine kinase inhibitors: structure-activity relationships for a series of 9-alkoxymethyl-12-(3-hydroxypropyl)indeno[2,1a]pyrrolo[3,4-c]carbazole-5-ones and the identification of CEP-5214 and its dimethylglycine ester prodrug clinical candidate CEP-7055. J. Med. Chem. 2003, 46, $5375-5388$.

[27] Mulholland, P. J.; Ferry, D. R.; Anderson, D.; Hussain, S. A., Young, A. M.; Cook, J. E.; Hodgkin, E.; Seymour, L. W.; Kerr, D. J. Pre-clinical and clinical study of QC12, a water soluble pro-drug of quercetin. Ann. Oncol. 2001, 12, 245-248.

[28] Warren, M. S.; Rautio, J. Prodrugs designed to target transporters for oral drug delivery. In: Prodrugs and targeted delivery-towards better ADME properties; Rautio, J., Ed.; Wiley-VCH Verlag \& Co. KgaA, Weinheim, 2011, pp 133-151.

[29] Beutner, K. R. Valacyclovir: a review of its antiviral activity, pharmacokinetic properties, and clinical efficacy. Antivir. Res. 1995, 28, 281-290 and references therein.

[30] Cocohoba, J. M.; McNicholl, I. R. Valganciclovir: an advance in cytomegalovirus therapeutics. Ann. Pharmacother. 2002, 6, 1075-1079 and references therein.

[31] Ganapathy, M. E.; Huang, W.; Ganapathy, V.; Leibach, F. H. Valacyclovir: a substrate for the intestinal and renal peptide transporters PEPT1 and PEPT2. Biochem. Bioph. Res. Co. 1998, 246, 470-475.

[32] Han, H.; de Vrueh, R. L. A.; Rhie, J. K.; Covitz, K. Y.; Smith, P. L.; Lee, C.; Oh, D.; Sadée, W.; Amidon, G. L. 5'-amino acid esters of antiviral nucleosides, acyclovir, and AZT are absorbed by the intestinal PEPT1 peptide transporter. Pharmacol. Res. 1998, 15, 1154-1159.

[33] Sugawara, M.; Huang, W.; Fei, Y. J.; Leibach, F. H.; Ganapathy, V.; Ganapathy, M. E. Transport of valgancyclovir, a ganciclovir prodrug, via peptide transporters PEPT1 and PEPT2. J. Pharm. Sci. 2000, 89, 781-789.

[34] Hatanaka, T.; Haramura, M.; Fei, Y. J.; Miyauchi, S.; Bridges, C. C.; P. S.; Ganapathy, Smith, S. B; Ganapathy, V.; Ganapathy, M. E. Transport of amino acid-based 
prodrugs by the $\mathrm{Na}+$ and $\mathrm{Cl}(-)$ coupled amino acid transporter ATB $(0,+)$ and expression of the transporter in tissues amenable for drug delivery. J. Pharmacol. Exp. Ther. 2004, 308, 1138-1147.

[35] Umapathy, N. S.; Ganapathy, V.; M. E. Ganapathy. Transport of amino acid esters and the amino-acid-based prodrug valgancyclovir by the amino acid transporter ATB $(0,+)$. Pharm. Res. 2004, 21, 1303-1310.

[36] Kim, I.; Chu, X. Y.; Kim, S.; Provoda, C. J.; Lee, K. D.; Amidon, G. I. Identification of a human valacyclovirase: biphenyl hydrolase-like protein as valacyclovir hydrolase. J. Biol. Chem. 2003, 278, 25348-25356.

[37] Pochopin, N. L.; Charman, W. N.; Stella, V. J. Amino acid derivatives of dapsone as water-soluble prodrugs. Int. J. Pharm. 1995, 121, 157-167.

[38] Pennick, M. Absorption of lisdexamfetamine dimesylate and its enzymatic conversion to D-amphetamine. Neuropsychriatr. Dis. Treat. 2010, 6, 317-327.

[39] Bundgaard, H.; Larsen, C.; Phorbek, P.; Prodrugs as drug delivery systems XXVI. Preparation and enzymatic hydrolysis of various water-soluble amino acid esters of metronidazole. Int. J. Phar. 1984, 18, 67-77.

[40] Bundgaard, H.; Larsen, C.; Arnold, E. Prodrugs as drug delivery systems XXVII. Chemical stability and bioavailability of a water-soluble prodrugs of metronidazole for parenteral administration. Int. J. Phar. 1984, 18, 79-87.

[41] Supko, J. G.; Malspeis, L. Dose-dependent pharmacokinetics of rapamycin-28-N, N-dimethylglycinate in the mouse. Cancer Chemoth. Pharm. 1994, 33, 325-330.

[42] Song, X.; Lorenzi, P. I.; Landowski, C. P.; Vig, B. S.; Hilfinger, J. M.; Amidon, G. I. Amino acid ester prodrugs of the anticancer agent gemcitabine: synthesis, bioconversion, metabolic bioevasion, and hPEPT1-mediated transport. Mol. Pharm. 2005, 2, 157-167.

[43] Balzarini, J.; Camarasa, M. J.; Velázquez, S. Prodrugs cleavable by CD26. US 10/555930 May 10, 2003.

[44] Fox, D. A.; Hussey, R. E.; Fitzgerald, K. A.; Acuto, O.; Poole, C.; Palley, L.; Daley, J. F.; Schossman, S. F.; Reinhert, E. L. Ta1, a novel 105 kDa human T cell activation antigen defined by a monoclonal antibody, J. Immunol. 1984, 133, 1250-1256.

[45] De Meester, I.; Vanhoof, G.; Lambeir, A. M.; Scharpe, S. CD26, let it cut or cut it down. Immunology Today 1999, 20, 367-375. 
[46] Lambeir, A. M.; Durinx, C.; Scharpe, S.; De Meester, I. Dipeptidyl-peptidase IV from bench to bedside: An update on structural properties, functions and clinical aspects of the enzyme DPPIV Crit. Rev. Cl. Lab. Sci. 2003, 40, 209-294.

[47] Yaron, A.; Naider, F. Proline-dependent structural and biological properties of peptides and proteins. Crit. Rev. Biochem. Mol. 1993, 28, 31-81.

[48] Mentlein, R. Dipeptidyl-peptidase IV (CD26)-role in the inactivation of regulatory peptides. Regul. Pept. 1999, 85, 9-24.

[49] Abbott, C.; McCaughan, G. W.; Baker, E.; Sutherland, G. R.; Genomic organization, exact localization, and tissue expression of the human CD26 (dipeptidyl peptidase IV) gene. Immunogenetics 1994, 40, 331-338.

[50] Aertgeerts, K.; Ye, S.; Tennant, M. G.; Kraus, M. L.; Rogers, J.; Sang, B.; Skene, R. J.; Webb D. R.; Prasad, G. S. Crystal structure of human dipeptidyl peptidase IV in complex with a decapeptide reveals details on substrate specificity and tetrahedral intermediate formation. Protein Science 2004, 13, 412-421.

[51] Oya, H.; Harada, M.; Nagatsu, T. Peptidase activity of glycylpropyl $\beta$ naphthylamidase from human submaxillary gland. Arch. Oral Biol. 1974, 19, 489-491.

[52] Püschel, G.; Mentlein, R.; Heymann, E. Isolation and characterization of dipeptidyl peptidase IV from human placenta. Eur. J. Biochem. 1982, 126, 359-365.

[53] Vanhoof, G.; Goossens, F.; De Meester, I.; Hendriks, D.; Scharpé, S. Proline motifs in peptides and their biological processing. Faseb J. 1995, 9, 736-744.

[54] Bouras, M.; Huneau, J.; Luengo, C.; Erlason-Albertsson, C.; Tomé, D. Metabolism of enterostatin in rat intestine, brain membranes, and serum: Differential involvement of proline-specific peptidases. Peptides 1995, 16, 399-405.

[55] Oravecz, T.; Pall, M.; Roderiquez, G.; Gorrell, M. D.; Ditto, M.; Nguyen, N. Y.; Boykins, R.; Unsworth, E.; Norcross, M. A. Regulation of the receptor specificity and function of the chemokine RANTES (Regulated on Activation, Normal T Cell Expressed and Secreted) by dipeptidyl peptidase IV (CD26)-mediated cleavage. J. Exp. Med. 1997, $186,1865-1872$.

[56] Proost, P.; De Meester, I.; Schols, D.; Struyf, S.; Lambeir, A. M.; Wuyts, A.; Opdenakker, G.; De Clercq, E.; Scharpé, S.; Van Damme, J. J. Amino-terminal truncation of chemokines by CD26/dipeptidyl-peptidase IV. Conversion of RANTES into a potent inhibitor of monocyte chemotaxis and HIV-1-infection. J. Biol. Chem. 1998, 273, 72227227. 
[57] Ohtsuki, T.; Hosono, O.; Kobayashi, H.; Munakata, Y.; Souta, A.; Shioda, T.; Morimoto, C. Negative regulation of the anti-human immunodeficiency virus and chemotactic activity of human stromal cell-derived factor 1 alpha by CD26/dipeptidyl peptidase IV. FEBS Lett. 1998, 431, 236-240.

[58] Proost, P.; Struyf, S.; Schols, D.; Durinx, C.; Wuyts, A.; Lenaerts, J. P.; De Clercq, E.; De Meester, I.; Van Damme, J. Processing by CD26/dipeptidyl-peptidase IV reduces the chemotactic and anti-HIV-1 activity of stromal-cell-derived factor $1 \alpha$. FEBS Lett. 1998, 432, 73-76.

[59] Su, J.; Andersson, E.; Horal, P.; Naghavi, M. H.; Palm, A.; Wu, Y. P. Eriksson, K.; Jansson, M.; Wigzell, H.; Svennerholm, B.; Vahlne, A. The nontoxic tripeptide glycylprolyl-glycine amide inhibits the replication of human immunodeficiency virus type 1. $J$. Human Virol. 2001, 4, 1-7.

[60] Su, J.; Naghavi, M. H.; Jejcic, A.; Furuta, Y.; Wu, Y. P.; Li, S.; Hall, W. W.; Goobar-Larsson, L.; Svennerholm, B.; Vahlne, A. The tripeptide glycyl-prolyl-glycine amide does not affect the early steps of the human immunodeficiency virus type 1 replication. J. Human Virol. 2001, 4, 8-15.

[61] Balzarini, J.; Andersson, E.; Schols, D.; Proost, P.; Van Damme, J.; Svennerholm, B.; Horal, P.; Vahlne, A. Obligatory involvement of CD26/dipeptidyl peptidase IV in the activation of the antiretroviral tripeptide glycylprolylglycinamide $\left(\mathrm{GPG}-\mathrm{NH}_{2}\right)$. Int. J. Biochem. Cell Biology. 2004, 36, 1848-1859.

[62] Balzarini, J.; Pérez-Pérez, M. J.; San-Félix, A.; Schols, D.; Perno, C. F.; Vandamme, A. M.; Camarasa, M. J.; De Clercq, E. 2',5'-Bis-O-(tert-butyldimethylsilyl)3'-spiro-5"-(4"-amino-1",2"-oxathiole-2",2"-dioxide)pyrimidine (TSAO) nucleoside analogues: Highly selective inhibitors of human immunodeficiency virus type 1 that are targeted at the viral reverse transcriptase. Proc. Natl. Acad. Sci. USA 1992, 89, 43924396.

[63] Camarasa, M. J.; Pérez-Pérez, M. J.; San-Félix, A.; Balzarini, J.; De Clercq, E. 3'Spironucleosides (TSAO derivatives), a new class of specific human immunodeficiency virus type 1 inhibitors: Synthesis and antiviral activity of 3'-spiro-5"-[4"-amino-1",2"oxathiole-2",2"-dioxide]pyrimidine nucleosides. J. Med. Chem. 1992, 35, 2721-2727.

[64] Camarasa, M. J.; San-Félix, A.; Velázquez, S.; Pérez-Pérez, M. J.; Gago, F.; Balzarini, J. TSAO compounds: The comprehensive story of a unique family of HIV-1 specific inhibitors of Reverse Transcriptase. Curr. Top. Med. Chem. 2004, 4, 945-963. 
[65] Camarasa, M. J.; Velázquez, S.; San-Félix, A.; Pérez-Pérez, M. J. Synthesis of 3'spiro-substituted nucleosides: chemistry of TSAO nucleoside derivatives. in Chemical Synthesis of nucleoside analogues, P. Merino (ed) John Wiley \& Sons, chapter 10, pages 427-472 (2013).

[66] De Castro, S.; Lobatón, E.; Pérez-Pérez, M. J.; San-Félix, A.; Cordeiro, A.; Andrei, G.; Snoeck, R.; De Clercq, E.; Balzarini, J.; Camarasa, M. J.; Velázquez, S. Novel [2',5'-Bis-O-(tert-butyldimethylsilyl)- $\beta$-D-ribofuranosyl]-3'-spiro-5"-(4"-amino$1 ", 2$ "-oxathiole-2",2"-dioxide) derivatives with anti-HIV-1 and anti-humancytomegalovirus activity. J. Med. Chem. 2005, 48, 1158-1168.

[67] Bonache, M. C.; Chamorro, C.; Velázquez, S.; De Clercq, E.; Balzarini, J.; Rodríguez Barrios, F.; Gago, F.; Camarasa, M. J.; San-Félix, A. Improving the antiviral efficacy and selectivity of HIV-1 Reverse Transcriptase inhibitor TSAO-T by the introduction of functional groups at the N-3 position. J. Med. Chem. 2005, 48, 66536660.

[68] García-Aparicio, C., Bonache, M. C., De Meester, I., San-Félix, A., Balzarini, J., Camarasa, M. J., Velázquez, S. Design and discovery of a novel dipeptidyl-peptidase IV (CD26)-based prodrug approach. J. Med. Chem. 2006, 49, 5339-5351.

[69] Umezawa, H.; Aoyagi, T.; Ogawa, K.; Hamada, M.; Takeuchi, T. Diprotins A and B, inhibitors of dipeptidyl aminopeptidase IV, produced by bacteria. J. Antibiot. 1984, 37, $422-425$.

[70] Schön, E.; Born, I.; Demuth, H. U.; Faust, J.; Neubert, K.; Steinmetzer, T.; Barth, A.; Ansorage, S. Dipeptidyl peptidase IV in the immune system. Effects of specific inhibitors on activity of dipeptidyl peptidase IV and proliferation of human lymphocytes. Biol. Chem. Hoppe Seyler 1991, 372, 305-311.

[71] Augustyns, K.; Bal, G.; Thonus, G.; Belyaev, A.; Zhang, X. M.; Bollaert, W.; Lambeir, A. M.; Durinx, C.; Goosens, F.; Haemers, A. The unique properties of dipeptidyl-peptidase IV (DPP IV/CD26) and the therapeutic potential of DPP IV inhibitors. Curr. Med. Chem. 1999, 6, 311-328.

[72] De Meester, I.; Lambeir, A. M.; Proost, P.; Scharpé, S. Dipeptidyl peptidase IV substrate. An update on in vitro peptide hydrolysis by human DPPIV. In: Dipeptidyl Aminopeptidases in Health and Disease. Hildebrandt, M.; Klapp, B.F.; Hoffman, T.; Demuth, H.-U., eds. Kluwer Academic/Plenum Publishers, New York, 2003, pp. 3-17. 
[73] García-Aparicio, C.; Diez-Torrubia, A.; Balzarini, J.; Lambeir, A. M.; Velázquez, S.; Camarasa, M. J. Efficient conversion of tetrapeptide-based TSAO prodrugs to the parent drug by dipeptidyl-peptidase IV (DPPIV/CD26). Antiviral Res. 2007, 76, 130-139.

[74] Diez-Torrubia, A.; García-Aparicio, C.; Cabrera, S.; De Meester, I.; Balzarini, J.; Camarasa, M. J.; Velázquez, S. Application of the dipeptidyl-peptidase IV (DPPIV/CD26)-based prodrug approach to different amine-containing drugs. J. Med. Chem. 2010, 53, 559-572.

[75] Brynes, P. J.; Bevilacqua, P.; Green, A. 6-aminoquinoline as a fluoregenic leaving group in peptide cleavage reactions. A new fluoregenic substrate for chymotrypsin. Anal. Biochem. 1981, 116, 408-413.

[76] Arcamone, F. Doxorubicine. New York, Academic Press, 1981.

[77] Weiss, R. B. The antracyclines: will we ever find a better doxorubicine? Semin. Oncol. 1992, 19, 670-686.

[78] Pérez-Pérez, M. J.; San-Félix, A.; Balzarini, J.; De Clercq, E.; Camarasa, M. J. TSAO-analogues. Stereospecific synthesis and anti-HIV-1 activity of 1-[2',5'-Bis-O-(tertbutyldimethylsilyl)- $\beta$-D-ribofuranosyl]-3'-spiro-5"-(4"-amino-1",2"-oxathiole-2" ,2"dioxide) pyrimidine and pyrimidine-modified nucleosides. J. Med. Chem. 1992, 35, 2988-2995.

[79] Rustum, Y. M.; Raymakers, R.A. 1-Beta-arabinofuranosylcytosine in therapy of leukemia: preclinical and clinical overview. Pharmacol. Ther. 1992, 56, 307-321.

[80] Adenine arabinoside: An Antiviral Agent. Pava-Langston, D.; Buchanan, R. A.; Alford, C. A. Eds.; Raven Press: New York, 1975.

[81] De Clercq, E. Antiherpes drugs: Promises and pitfalls. Eur. J. Microbiol. Infect. Dis. 1984, 3, 96-107.

[82] Superti, F.; Ammendolia, M.G.; Marchetti, M. New advances in anti-HSV chemotherapy. Curr. Med. Chem. 2008, 15, 900-911.

[83] Diez-Torrubia, A.; Cabrera, S.; Lambeir, A. M.; Balzarini, J.; Camarasa, M. J.; Velázquez, S. Dipeptidyl peptidase IV (DPPIV/CD26)-based progrugs of hydroxycontaining drugs, ChemMedChem. 2012, 7, 618-628.

[84] Faulds, D.; Brogden, R. N. Didanosine- a review of its antiviral activity, pharmacokinetic properties and therapeutic potential in human-immunodeficiency-virus infection. Drugs, 1992, 44, 94-116. 
[85] Hansson, L.; Werkö, L. Beta-adrenergic blockade in hypertension. Am. Heart J. 1977, 93, 394-402.

[86] Pommier, Y. Topoisomerase I inhibitors: camptothecins and beyond. Nat. Rev. Cancer 2006, 6, 789-802.

[87] Schaeffer, H. J.; Beauchamp, L.; De Miranda, P.; Elion, G. B.; Bauer, D. J.; Collins, P. 9-(2-Hydroxyethoxymethyl)guanine activity against viruses of the herpes group, Nature, 1978, 272, 583-585.

[88] de Miranda, P.; Blum, M. R. Pharmacokinetics of acyclovir after intravenous and oral administration. J. Antimicrob. Chemother. 1983, 12 (Suppl. B), 29-37.

[89] De Clercq, E.; Field, H. J. Antiviral prodrugs-the development of successful prodrug strategies for antiviral chemotherapy, Br.J. Pharmacol. 2006, 147, 1-11.

[90] Gao, H.; Mitra, A. K. Synthesis of Acyclovir, Ganciclovir and Their Prodrugs: A Review, Synthesis-Stuttgart, 2000, 3, 329-351.

[91] Karaman, R.; Dajani, K. K.; Qtait, A.; Khamis, M. Prodrugs of acyclovir-a computational approach, Chem. Biol. Drug Des. 2012, 79, 819-834 and references therein.

[92] Santos, C. R.; Capela, R.; Pereira, C. S. G. P., Valente, E.; Gouveia, L.; Pannecouque, C.; De Clercq, E.; Moreira, R.; Gomes, P. Structure-activity relationships for dipeptide prodrugs of acyclovir: Implications for prodrug design. Eur. J. Med. Chem. 2009, 44, 2339-2346.

[93] Colla, L.; De Clercq, E.; Busson, R.; Vanderhaeghe, H. Synthesis and antiviral activity of water-soluble esters of acyclovir [9-[(2-hydroxyethoxy)methyl]guanine]. $J$. Med.Chem. 1983, 26, 602-604;

[94] Beauchamp, L. M.; Krenitsky, T. A. Acyclovir prodrugs: the road to valacyclovir. Drugs Fut. 1993, 18, 619-628.

[95] Vigil, K. J.; Chemaly, R. F. Valacyclovir: approved and off-label uses for the treatment of herpes virus infections in immunocompetent and immunocompromised adults. Exp. Opin. Pharmacother. 2010, 11, 1901-1913 and references therein.

[96] Diez-Torrubia, A.; Cabrera, S.; de Castro, S.; García-Aparicio, C.; Mulder, G.; De Meester, I.; Camarasa, M. J.; Balzarini, J.; Velázquez, S. Novel water-soluble prodrugs of acyclovir cleavable by the dipeptidylpeptidase IV (DPP IV/CD26) enzyme. Eur. J. Med. Chem., 2013, 70, 456-468.

[97] McGuigan, C.; Yarnold, C. J.; Jones, G.; Velázquez, S.; Barucki, H.; Brancale, A.; Andrei, G.; Snoeck, R.; De Clercq, E.; Balzarini, J. Potent and selective inhibition 
of Varicella-Zoster Virus (VZV) by nucleoside analogues with an unusual bicyclic base. J. Med. Chem. 1999, 42, 4479-4484.

[98] McGuigan, C.; Barucki, H.; Blewet, S.; Carangio, A.; Erichsen, J. T.; Andrei, G.; Snoeck, R.; De Clercq, E.; Balzarini, J. Highly potent and selective inhibition of varicella-zoster virus by bicyclic furopyrimidine nucleosides bearing an aryl side chain. $J$. Med. Chem. 2000, 43, 4993-4997.

[99] Mc Guigan, C.; Balzarini, J. Aryl furano pyrimidines; The most potent and selective anti-VZV agents reported to date. Antiviral Res. 2006, 71, 149-153.

[100] McGuigan, C.; Pathirana, R. N.; Migliore, M.; Adak, V.; Luoni, G.; Jones, A. T.; Diez-Torrubia, A.; Camarasa, M. J.; Velázquez, S.; Henson, G.; Snoeck, R.; Andrei, G.; Balzarini, J. Preclinical development of bicyclic nucleoside analogues as potent and selective inhibitors of varicella zoster virus. J. Antimicrob. Chemother. 2007, 60, 13161330.

[101] Diez-Torrubia, A.; Balzarini, J.; Andrei, G.; Snoeck, R.; De Meester, I.; Camarasa, M. J.; Velázquez, S. Dipeptidyl-peptidase IV-dependent water-soluble prodrugs of highly lipophilic bicyclic nucleoside analogues. J. Med. Chem. 2011, 54, 1927-1942.

[102] Diez-Torrubia, A.; Cabrera, S.; De Meester, I.; Camarasa, M. J.; Balzarini, J.; Velázquez, S. Dipeptidyl-peptidase IV-activated prodrugs of anti-Varicella Zoster Virus bicyclic nucleoside analogues containing different self-cleavage spacers systems. ChemMedChem. 2012, 7, 1612-1622.

[103] For selected examples of prodrugs bearing these type of diamine cyclization spacers, see for example references 103-105: De Groot, F. M. H.; Van Berkom, L. W. A.; Scheeren, H. W. Synthesis and biological evaluation of 2'-carbamate-linked and 2'carbonate-linked prodrugs of paclitaxel: selective activation by the tumor-associated protease plasmin. J. Med. Chem. 2000, 43, 3093-3102.

[104] Saari, W. S.; Schwering, J. E.; Lyle, P. A.; Smith, S. J.; Engelhardt, E. L. Cyclization-activated prodrugs. Basic carbamates of 4-hydroxyanisole. J. Med. Chem. 1990, 33, 97-101.

[105] Dipeptides have been widely used as drug carriers for cyclization-activated prodrugs, see for example references 106-110: Santos, C.; Mateus, M. L.; Santos, A. P.; Moreira, R.; Oliveira, E.; Gomes, P. Cyclization-activated prodrugs. Synthesis, reactivity and toxicity of dipeptide esters of paracetamol. Bioorg. Med. Chem. Lett. 2005, 15, 15951598. 
[106] Santos, C.; Morais, J.; Gouveia, L.; De Clercq, E.; Pannecouque, C.; Nielsen, C. U.; Steffansen, B.; Moreira, R.; Gomes, P. Dipeptide derivatives of AZT: synthesis, chemical stability, activation in human plasma, hPEPT1 affinity and antiviral activity. ChemMedChem 2008, 3, 970-978.

[107] Tsume, Y.; Hilfinger, J. H.; Amidon, G. L. Enhanced cancer cell growth inhibition by dipeptide prodrugs of floxuridine: increased transporter affinity and metabolic stability. Mol. Pharmaceut. 2008, 5, 717-727.

[108] Jain, R.; Duvvuri, S.; Kansara, V.; Mandava, N. S. Mitra, A.K. Intestinal absorption of novel-dipeptide prodrugs of saquinavir in rats. Int. J. Pharm. 2007, 336, 233-240 and references therein.

[109] Talluri, R. S.; Samanta, S. K.; Gaudana, R.; Mitra, A. K. Synthesis, metabolism and cellular permeability of enzymatically stable dipeptide prodrugs of acyclovir. Int. $J$. Pharm. 2008, 361, 118-124.

[110] Capasso, S.; Vergara, A.; Mazzarella, L. Mechanism of 2,5-dioxopiperazine formation. J. Am. Chem. Soc. 1998, 120, 1990-1995.

[111] Keese, R.; Meyer, M. The structural basis of the geminal-dimethyl effect. Tetrahedron 1993, 49, 2055-2064.

[112] Ramachandran, G. N.; Mitra, A. K. An explanation for the rare ocurrence of cis peptide units in proteins and polypeptides. J. Mol. Biol. 1976, 107, 85-92. 